\title{
HYDROLOGIC DATA FOR THE VAMOOSA AQUIFER, EAST-CENTRAL OKLAHOMA
}

\section{U. S. GEOLOGICAL SURVEY}

Open- File Report 77-487
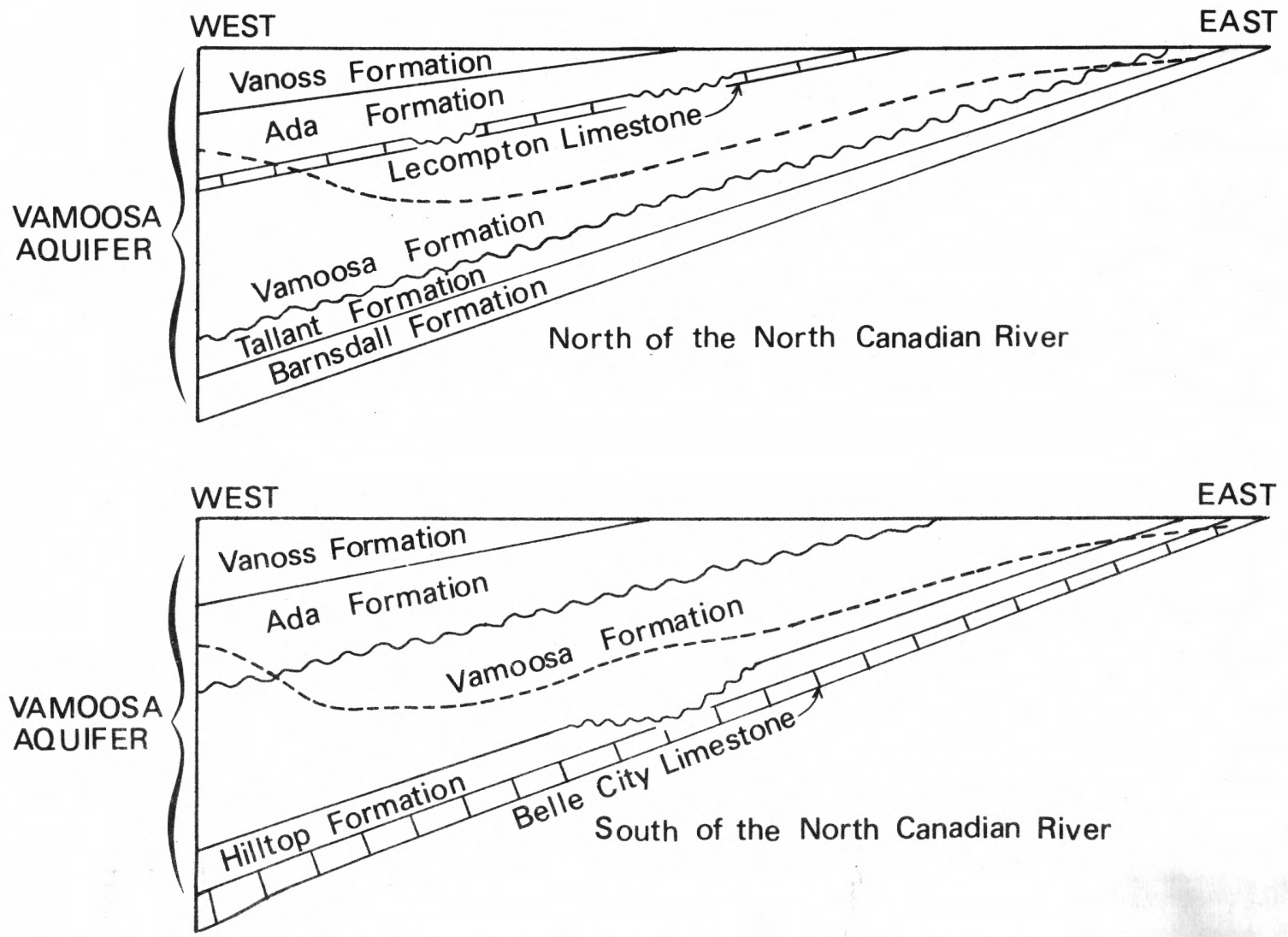

- - - Base of water containing less than $1500 \mathrm{mg} / \mathrm{L}$ dissolved solids

Prepared in cooperation with the OKLAHOMA GEOLOGICAL SURVEY

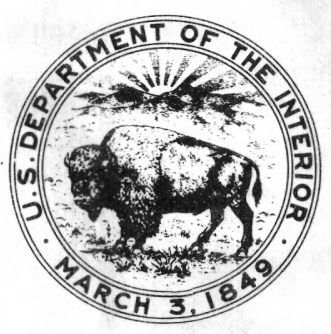




\section{UNITED STATES \\ DEPARTMENT OF THE INTERIOR \\ Geological Survey}

\section{HYDROLOGIC DATA FOR THE VAMOOSA AQUIFER, EAST-CENTRAL OKLAHOMA \\ By Joseph J. D'Lugosz and Roger G. McClaflin}

Open File Report 77-487

Prepared in cooperation with the OKLAHOMA GEOLOGICAL SURVEY

Oklahoma City, Oklahoma

; July 1977 

Introduction................................. 2

Selected references............................... 4

Explanation of information in the tables................. 9

Conversion factors.............................. 10

\section{ILLUSTRATIONS}

Figure 1.--Map showing the location of the study area.......... 1

Figure 2.--Hydrologic data for Hilliby Creek basin........... 11

Figure 3.--Hydrologic data for Polecat Creek basin............ 12

Figure 4.-- Well hydrographs and monthly precipitation......... 13

\section{TABLES}

Table 1.--Records of selected wells.................... 14

Table 2.--Chemical analyses of water from selected wells....... 28

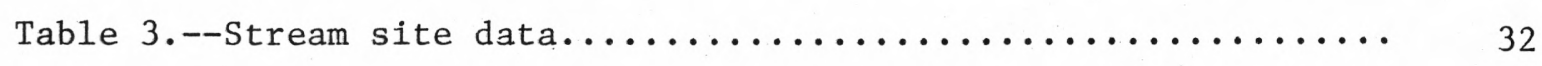





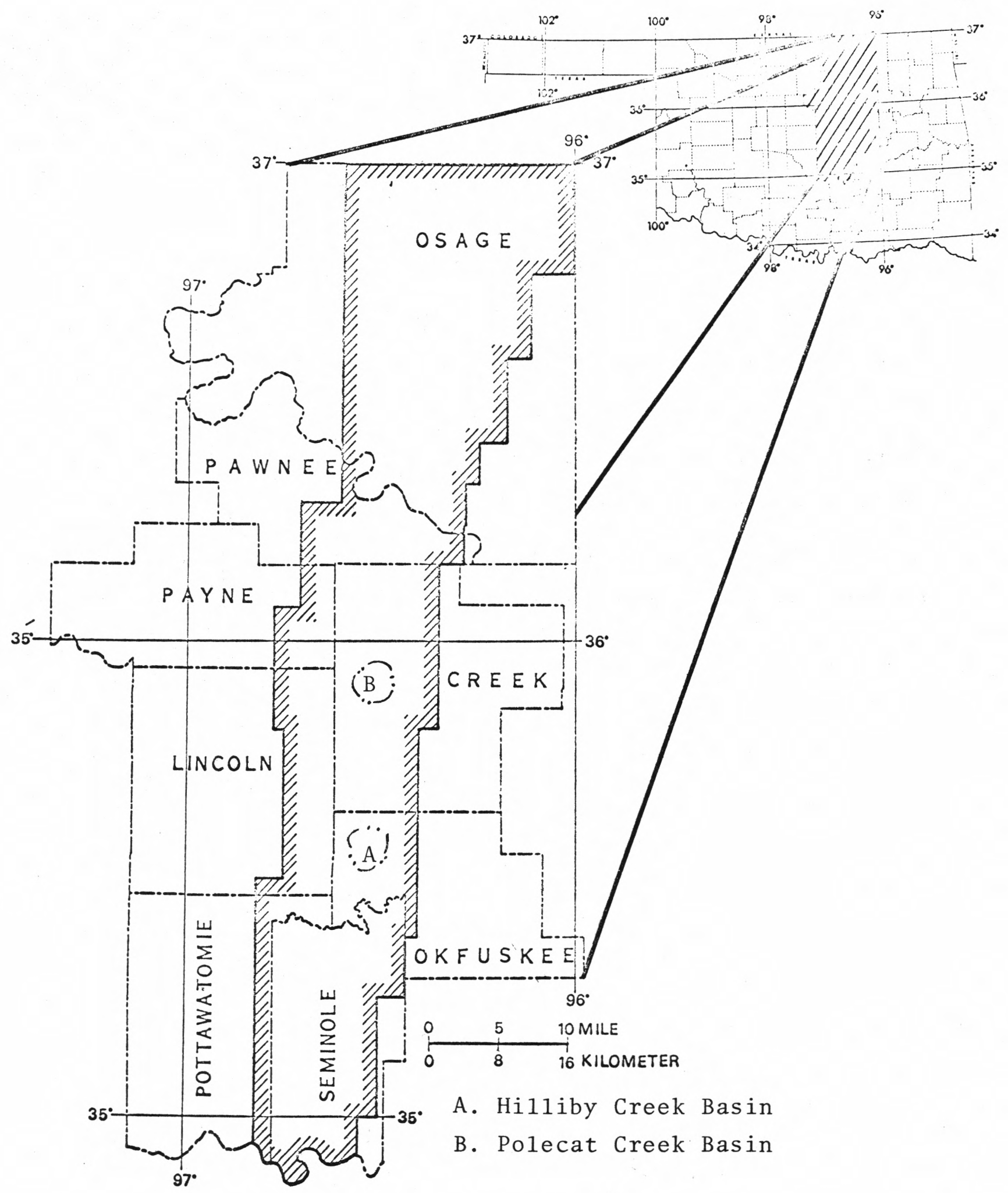

Figure 1.--Location of the study area. 
Hydrologic data for the Vamoosa aquifer, east-central oklahoma

by

Joseph J. D'Lugosz and Roger G. McClaf1in

\section{Introduction}

Most of the data presented in this report were collected by the U.S. Geological Survey as part of a study of the geohydrology of the Vamoosa aquifer, in east-central 0klahoma, in cooperation with the Oklahoma Geological Survey. Some of the data listed in the tables were collected by Bingham and Moore (1975) and Bingham and Bergman (in press). Acknowledgment is extended to city officials and individuals who provided the data for this report. 
The standard method of giving location by fractional section, section, township, and range is replaced by the method illustrated in the diagram below. The location of the site indicated by the dot normally would be described as NiN $1 / 4 \mathrm{SE} 1 / 4 \mathrm{SW} 1 / 4 \mathrm{sec} .6, \mathrm{~T} .15 \mathrm{~N} ., \mathrm{R} .08 \mathrm{E}$. The method used in this report reverses the order and indicated quarter subdivisions of the section by letters. By this method the location of the site is given as $15 \mathrm{~N}-08 \mathrm{E}-06 \mathrm{CDB} 1$. The final digit (1) is the sequential number of a site within the smallest fractional subdivision. Some of the sites are located only to the nearest section or quarter section.

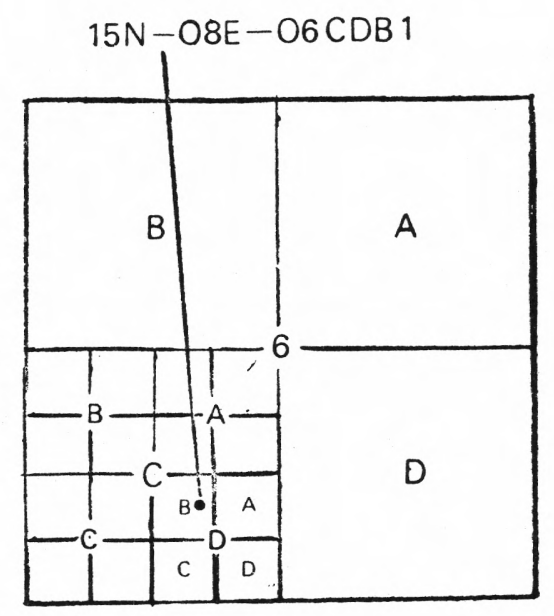


Bass, N.W., Kennedy, L.E., Dillard, W.R., Reese, William, Leatherock, Otto, Hengst, J.H., 1938, Subsurface geology and oil and gas resources of Osage County, Oklahoma, Pt. 1, Townships 22 and 23 North, Ranges 10 and 11 East: U.S. Geol. Survey Bull. 900-A, p. $1-45$.

Bass, N.W., Kennedy, L.E., Dillard, W.R., Reese, William, Leatherock, Otto, Hengst, J.H., 1939, Subsurface geology and oil and gas resources of Osage County, Oklahoma, Pt. 3, Townships 24 and 25 North, Ranges 8 and 9 East: U.S. Geol. Survey Bull. 900-C, p. 88-129.

Beckwith, H.T., 1928, Oil and gas in Oklahoma; geology of Osage County: Oklahoma Geol. Survey Bu11. 40-T, 63 p. Berryhi11, R.A., 1961, Subsurface geology of south-central Pawnee County, Oklahoma: Oklahoma City Geol. Soc. Shale Shaker, v. 12, no. 41, p. $2-18$

Bingham, R.H. and Moore, R.L., 1975, Reconnaissance of the water resources of the Oklahoma City quadrangle, central Oklahoma: Oklahoma Geol. Survey Hydrol. Atlas 4, 4 maps.

Bingham, R.H. and Bergman, D.L., 1978, Reconnaissance of the water resources of the Enid quadrangle: Oklahoma Geol. Survey Hydrol. Atlas 7, 4 maps (in press). 
Branson, C.C., 1956, Pennsylvanian history of northeastern Oklahoma:

Tulsa Geo1. Soc. Digest, v. 24, p. 83-86.

Brensing, D.G., Oakes, Harvey, Scriven, David, Talley, E.C., Mikles, H.P.,

Thorp, James, 1959, Soil Survey of Creek County, Oklahoma: U.S.

Dept. of Agriculture, Soil Conservation Service, Series 1950, no. $5,43 \mathrm{p}$.

Buckhannan, W.H., Bogard, V.A., Bush, H.H., Carson, A.D., Graham, E.0., Sparwasser, W.A., Walker, G.D., Carter, W.T., 1952, Soil Survey of Okfuskee County, Oklahoma: U.S. Dept. of Agriculture, Soil Conservation Service, Series 1940, no. 7, 110 p.

Burwell, A.L., 1942, The possibility of magnesia from Oklahoma oil

field brines: Oklahoma Geol. Survey Mineral Report 14, 14 p. Chenoweth, P.A., 1959, Sources of the Vamoosa quartzite pebbles:

Oklahoma Geol. Survey Oklahoma Geology Notes, v. 19, no. 11, p. 229-232.

Collins, A.G., and Egleson, G.C., 1967, Iodide abundance in oil-field brines in Oklahoma: Science, v. 156, no. 3777, p. 934-935. Cutolo-Lotano, Francisco, 1969, Subsurface geology of the Seminole area, Seminole, Pottawatomie, and Okfuskee Counties, Oklahoma: Oklahoma City Geol. Soc. Shale Shaker, v. 19, no. 7, p. 118-130. Fambrough, J.W., 1963, Isope - h and lithofacies study of Virgilian and Missourian series of north-central Oklahoma: Oklahoma City Geol. Soc. Shale Shaker, v. 13, no. 5, p. 2-8, 10-12, 14-23, 26. Foley, L.L., 1926, The origin of the faults in Creek and Osage Counties Oklahoma: Am. Assoc. Petroleum Geologists Bull., v. 10, no. 3, p. 293-303. 
Fronjosa, Ernesto, 1965, A study of Okalhoma water flood statistics: Oklahoma Univ. unpub. M.S. thesis, 87 p.

Galloway, H.M., Templin, E.H., Oakes, Harvey, 1959, Soil Survey of Pawnee County, Oklahoma: U.S. Dept. of Agriculture, Soil Conservation Service, Series 1952, no. 4, 71 p.

Greig, P.B., Jr., 1950, Geology of Pawnee County, Oklahoma: Oklahoma Geo1. Survey Bu11. 83, 188 p.

Hart, D.L., Jr., 1974, Reconnaissance of the water resources of the Ardmore and Sherman quadrangles, southern Oklahoma: Oklahoma Geol. Survey, Hydro1. Atlas 3, 4 maps.

Ickes, E.L., 1926, Origin of the faults in Creek and Osage Counties, Oklahoma: Am. Assoc. Petroleum Geologists Bu11., v. 10, no. 7, p. $722-729$.

Kennedy, L.E., 1940, Subsurface geology and oil and gas resources of Osage County, Oklahoma: U.S. Geo1. Survey Bu11. 900-D, part 4, p. $131-172$.

Kirk, M.S., 1957, A subsurface section from Osage County to Okfuskee County, Oklahoma: Oklahoma City Geol. Soc. Shale Shaker, v. 7, no. 6, p. 204 .

Koschmann, A.H., 1928, Oil and gas in Oklahoma; geology of Payne County: Oklahoma Geo1. Survey Bull. 40-X, 13 p.

Kramer, W.B., 1939, Enechelon faults in Oklahoma: Am. Assoc. Petroleum Geologists Bul1. v. 18, no. 2, p. 243-248.

Levorson, A.I., 1928, Oil and gas in Oklahoma, Geology of Seminole County: Oklahoma Geol. Survey Bull. 40-BB, 70 p. 
Masters, K.E., 1957, Geology of the Prague area Lincoln and Pottawatomie Counties, Oklahoma: Oklahoma City Geol. Soc. Shale Shaker, Digest II, v. VI-VIII.

Morgane11i, Danie1, 1973, Depositional environment and trend of uppermost part of the Vamoosa Formation and Lecompton Limestone in the eastern part of north-central Oklahoma: Oklahoma State Univ. unpub. M.S. thesis, 68 p.

Oakes, M.C., 1959, Geology anḍ mineral resources of Creek County, Oklahoma: Oklahoma Geol. Survey Bull. 81, 134 p.

Oklahoma Water Resources Board, 1971, Appraisal of the water and related land resources of Oklahoma, (Region 8): Oklahoma Water Resources Board pub., no. 34, 141 p.

--- , 1971, Appraisal of the water and related land resources of Oklahoma, (Region 9): Oklahoma Water Resources Board pub. 36, 149 p.

---- , 1972, Appraisal of the water and related 1and resources of Oklahoma, (Region 10): Oklahoma Water Resources Board pub. 40, $137 \mathrm{p}$.

Powers, Sidney, 1927, The Seminole uplift Oklahoma: Am. Assoc. Petroleum Geologists Bul1., v. 11, no. 10, p. 1097-1108.

Ries, R.E., 1954, Geology and mineral resources of Okfuskee County, Oklahoma: Oklahoma Geol. Survey Bul1. 77, 120 p. Sherrill, R.E., 1929, Origin of the en echelon faults in north-central Oklahoma: Am. Assoc. Petroleum Geologists Bull., v. 13, no. 10, p. $31-37$.

Tanner, U.F., 1956a, Geology of north-eastern Osage County, Ok1ahoma: Oklahoma Geol. Survey Circ. 40, 76 p. 
--_- , 1956b, Geology of Seminole County, Oklahoma: Okalhoma Geol. Survey Bu11. 74, 175 p.

Terrel1, D.M., 1972, Trend and genesis of the Pennsylvanian Elgin Sandstone in the western part of north eastern Oklahoma: Oklahoma State Univ. unpub. M.S. thesis, 79 p.

U.S. Geological Survey, 1974, Water resources data for Oklahoma: Part 1, Surface water records, $194 \mathrm{p}$.

Williams, G.E. and Bartolina, D.G., 1970, Soil survey of Lincoln County, Oklahoma: U.S. Dept. of Agriculture, Soil Conservation Service, $57 \mathrm{p}$. 
Explanation of Information in the Tables

(Well Depth) Depth of the well below land surface to the nearest foot. Numbers following the depth of the well are accuracy codes as follows:

0. Accurate within 1 foot

1. Less than 1 foot

5. Estimated

6. Reported

(Water Use) Water use is coded as follows:

C. Commercial

H. Domestic

I. Irrigation

P. Public supply

S. Stock supply

U. Unused

(Depth to water) Depth to the water level below land surface. Letters following the water level are accuracy codes as follows:

A. Tape - better than 1 foot

B. Tape - less than 1 foot

G. Reported 
(Aquifer Code) The Vamoosa aquifer consists of the Vamoosa Formation and all underlying and overlying formations which are lithologically similar and are hydrologically connected. The individual codes are as follows:

(Aquifers)--

Informal Terms - OA Alluvium

OT Terrace deposits

Pennsylvanian - DA Vanoss Formation

DB Ada Formation

DE Vamoosa Formation

(Remarks) The code used is as follows:

E. - Electric $\log$

D. - Driller's log

J. - Gamma ray $\log$

Q. - Water-quality data available

R. - Radiation 1ogs

S. - Spring

BR. - Bromide concentration in $\mathrm{mg} / \mathrm{L}$

INJ. - Injection wel1

\section{Conversion Factors}

English units used in this report may be converted to metric units by the following conversion factors:

Multiply English unit

Inches (in)

Feet ( $f t)$

Gallons per minute (gal/min)

Cubic feet per second $\left(\mathrm{ft}^{3} / \mathrm{s}\right)$
By

25.4

.3048

.06309

.02932
To obtain Metric unit

Millimeters (mm)

Meters (m)

Liters per second (L/s)

Cubic meters per second $\left(\mathrm{m}^{3} / 2\right)$ 


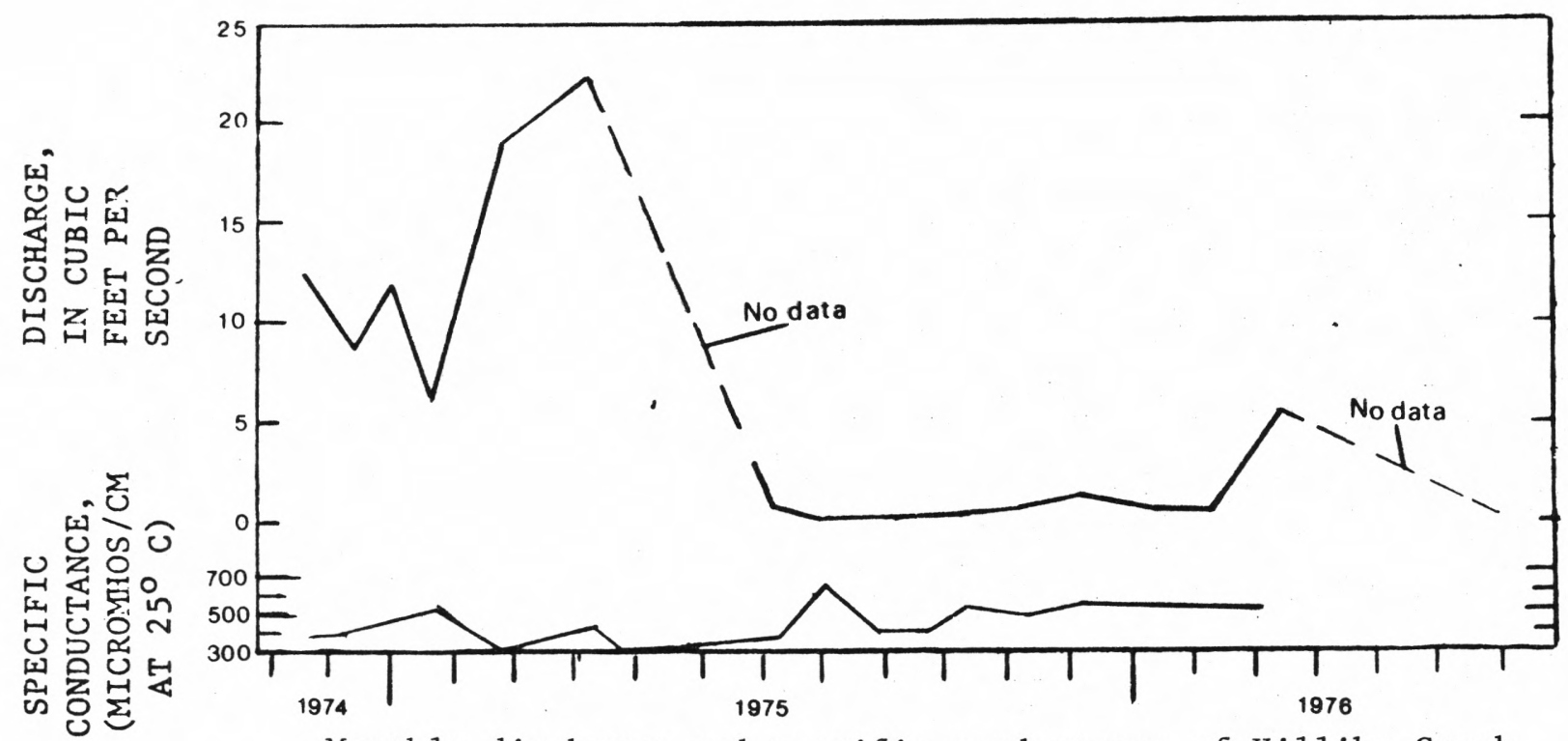

Monthly discharge and specific conductance of Hilliby Creek

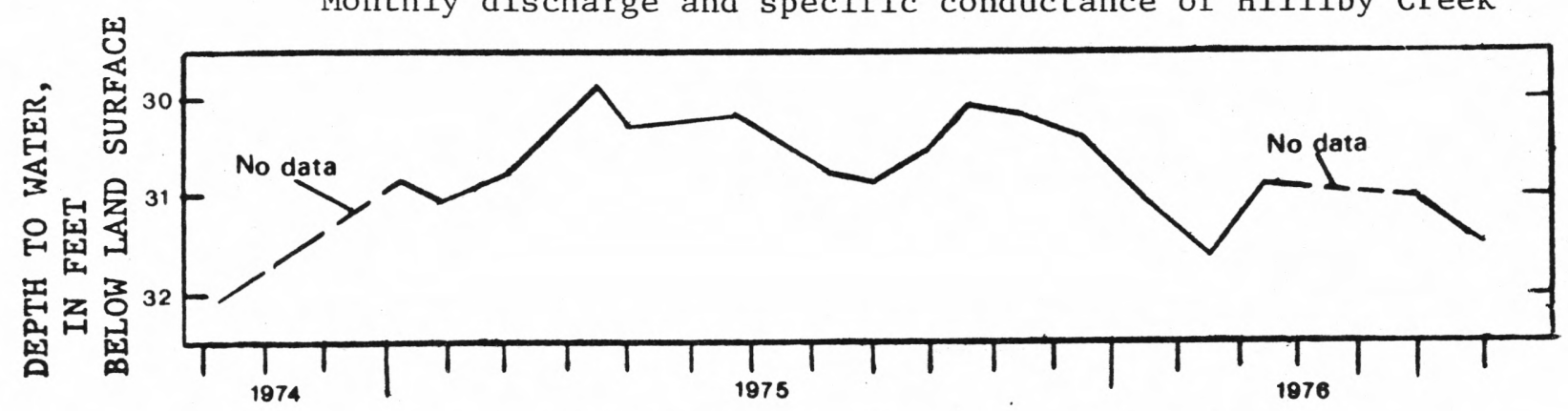

Average monthly water level in seven wells

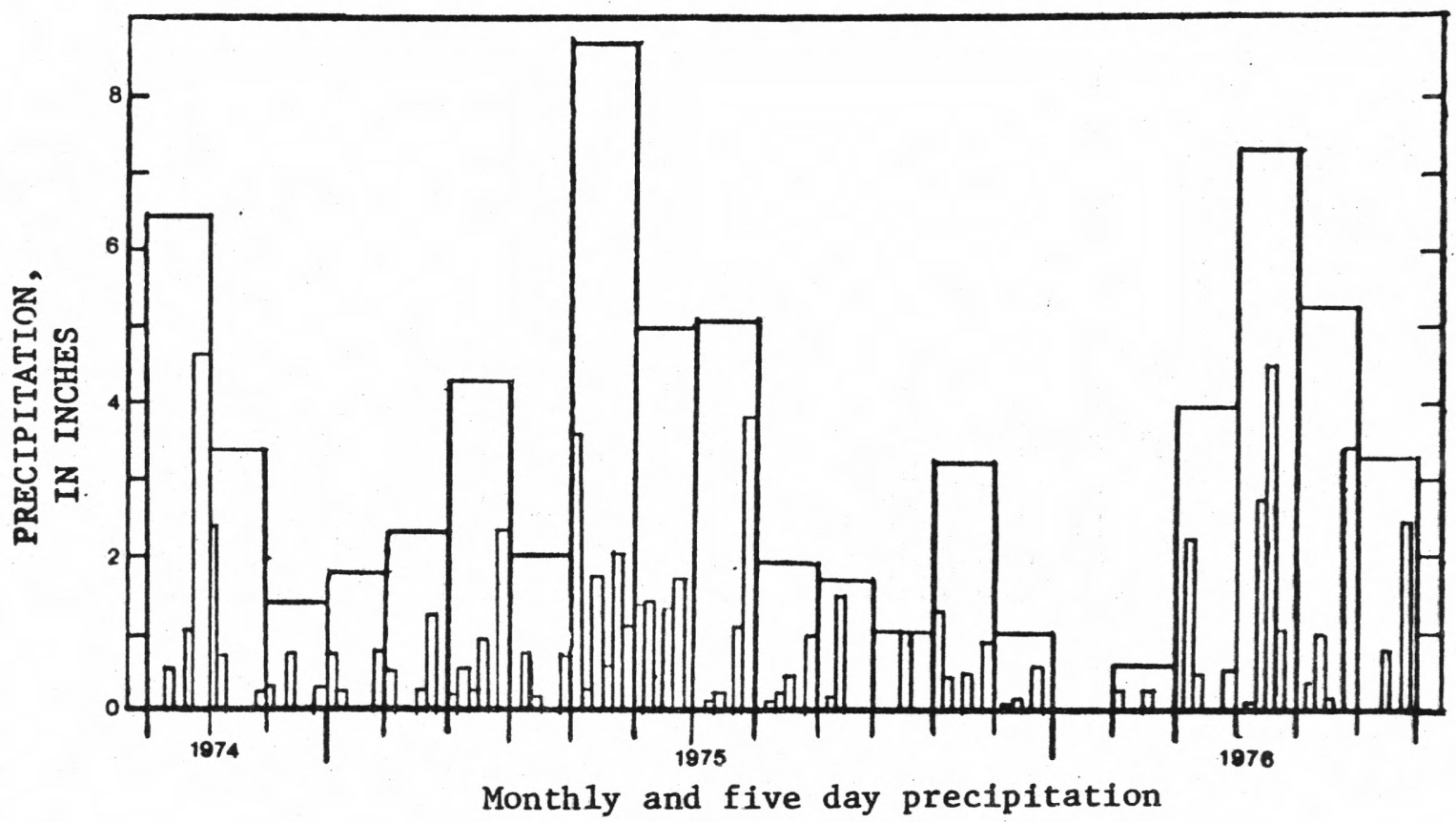

Figure 2.--Hydrologic data for Hilliby Creek basin 


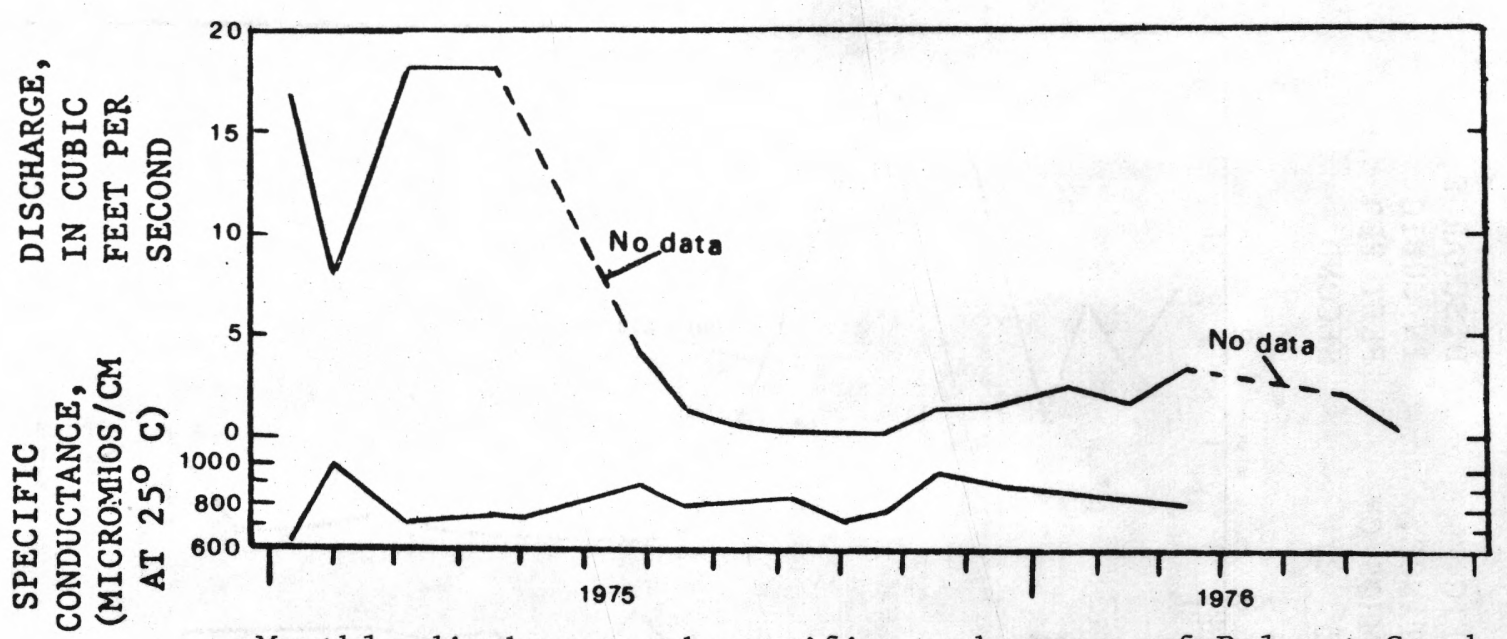

Month1y discharge and specific conductance of Polecat Creek

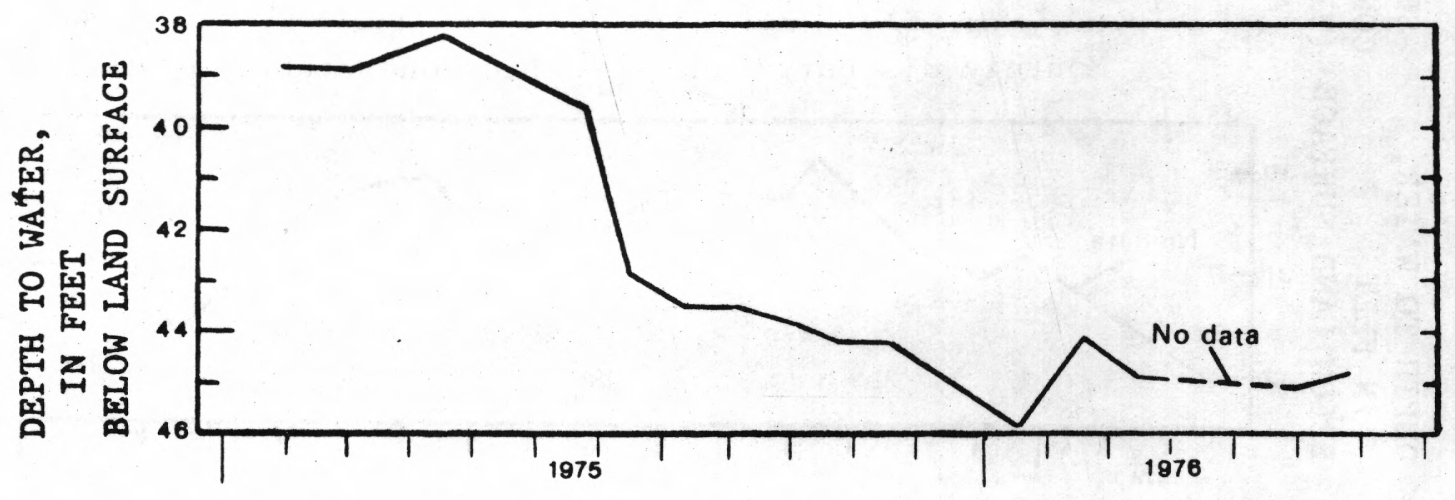

Average monthly water levels in three wells

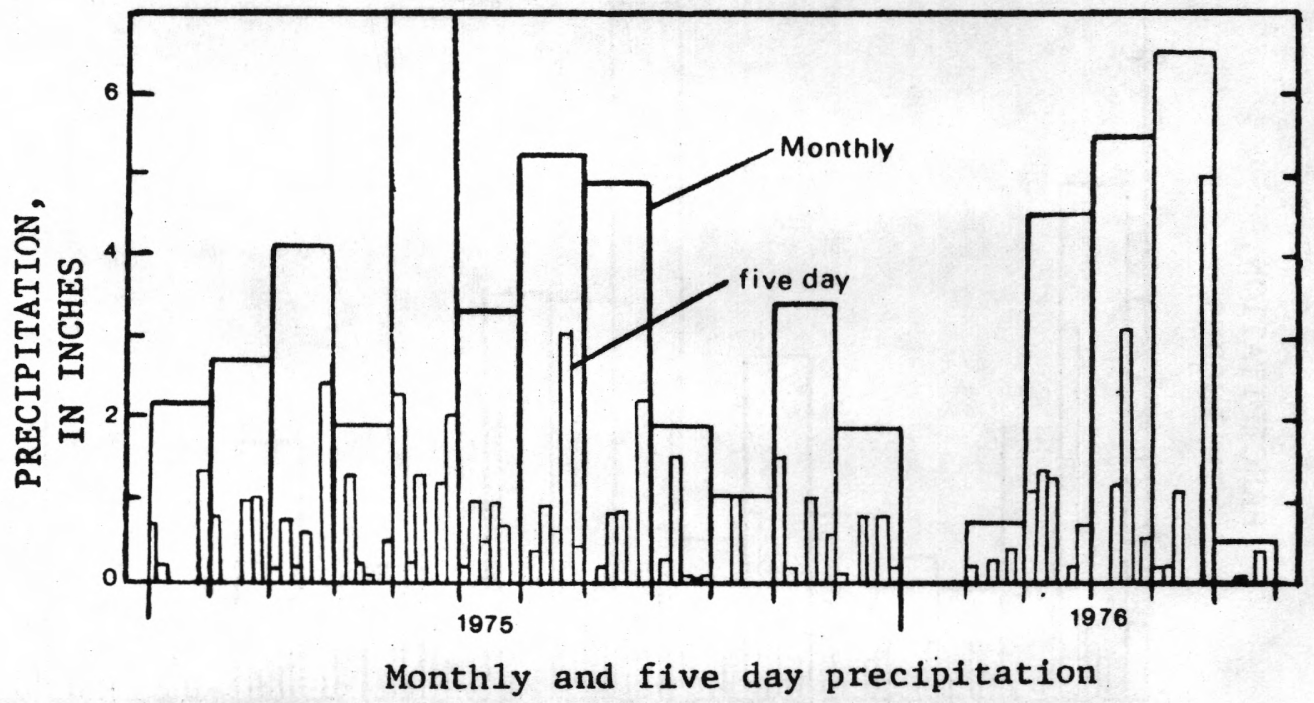

Figure 3.--Hydrologic data for Polecat Creek basin 

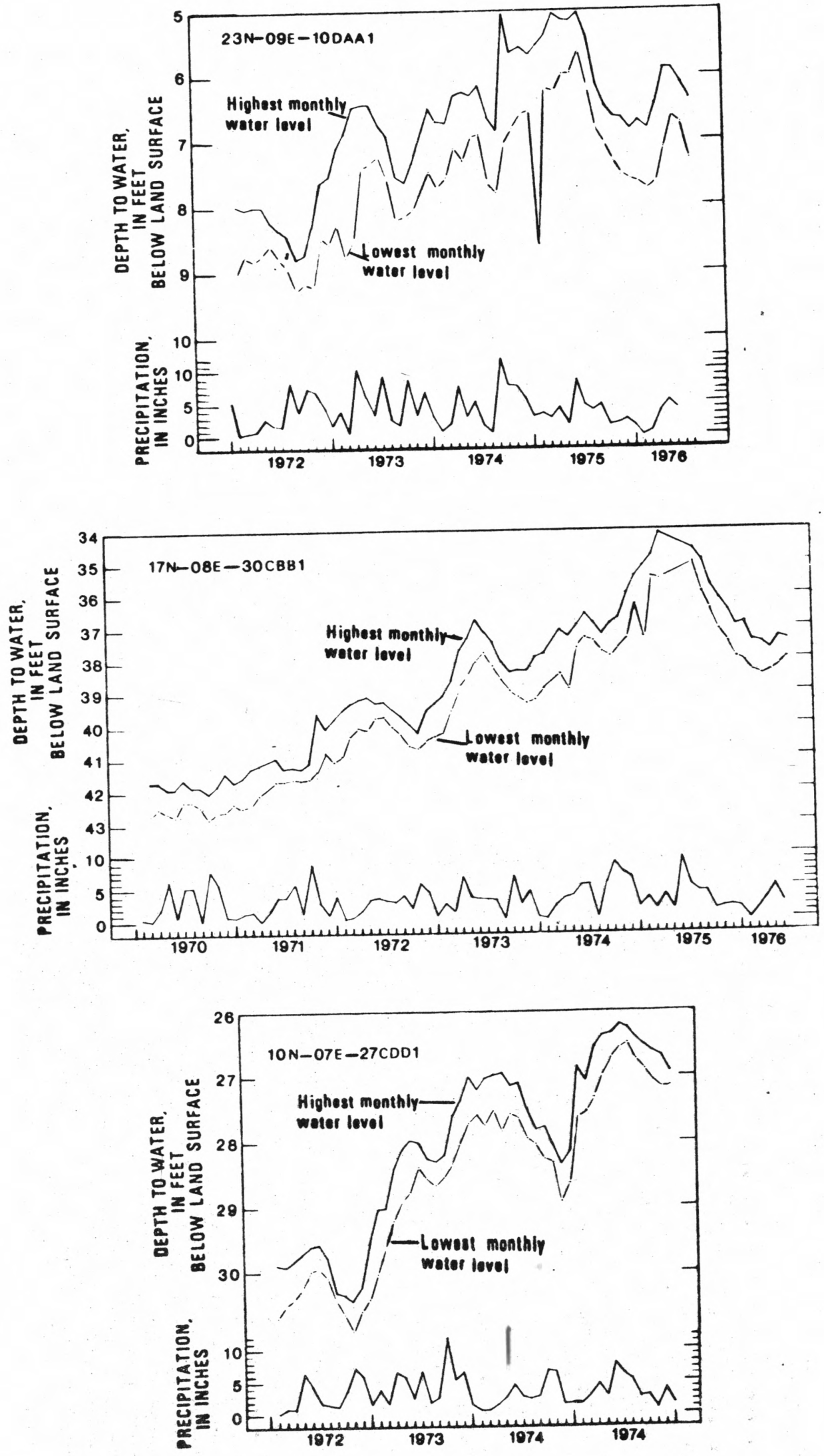

Figure 4.--We11 hydrographs and monthly precipitation 
Table 1.--Records of selected wells.

SEMINOLE COUNTY

\begin{tabular}{|c|c|c|c|c|c|c|c|c|c|c|c|}
\hline & DUNER & & & & & DEPTH & & & & ALTITUDE & \\
\hline WELL & UR & YEAR & WELL & WAIER & WELL & TO & DATE & WELL & & DF LANU & \\
\hline LUCATIUN & USER & DRILLED & $\begin{array}{l}\text { DEPTH } \\
\text { (FT) }\end{array}$ & USE & $\begin{array}{l}\text { OIAME TER } \\
\text { (IN) }\end{array}$ & $\begin{array}{l}\text { HATER } \\
(F \mid)\end{array}$ & $\begin{array}{l}\text { MEASURED } \\
(M U-Y R)\end{array}$ & $\begin{array}{c}\text { YIELU } \\
\text { (GAL/MIN) }\end{array}$ & AQUIFERS & $\begin{array}{c}\text { SURF ACE } \\
\text { (FT) }\end{array}$ & REMARKS \\
\hline
\end{tabular}

OSN-OSE-10BSB

$05 N-05 E-1086 \mathrm{~B} \quad 1$ $05 N-05 E-12 A B B$ $05 N-05 E-250 A D \quad 1$ OSN-OSE-OLBDC 1 $O O N-O S E-10 C C O 1$ $06 N-05 E-11 \mathrm{BAE}$ (OGN- DSE-11CDD $0 O N-0 S E-2 h C B B$ $0 G N-05 E-26 U D B$ OGN-OSE-2TUAA $06 N-06 E-07 D A D$

$\quad 0 O N-06 E-1 U A A O$

OOH-OGE-12ACC 1 $0 B N-06 E-1610001$ $06 N-06 E-25 C C C 1$ $07 \mathrm{~N}-05 \mathrm{E}-02 \mathrm{CCH} 1$ $07 N-05 E-120 D C 1$ $07 N-05 E-13 A C C 1$ $07 N-05 E-220001$ $07 N-05 E-26 H E B \quad 1$ $07 N-1) 5 E-27 A A A$ $07 H-00 E-O S C D D 1$ $07 N-06 E-13 B C A \quad 1$ $07 N-06 E-16 C C D$ $07 N-06 E-23 B C B 1$ $O 7 N-O B E-31 A D D 1$ $07 N-0 O E-3.3 B O U$ 0) $7 N-06 E-34 D C O \quad 1$ $07 N-07 E-05 A B B 1$ $08 N-05 E-1$ UDOD 1 $O B N-0 S E-11 A A U$ $0 B N-05 E-1$ SONOD 1 $08 N-05 E-2431) A$ $0 B N-05 E-360 A A 1$ $0 B N-0 O E-03 U D C 1$ 1) $B N=0 B E-07 C(D)$ $0 B N-0 O E-0700 C 1$

OHIN-OOEE- OUDODI 1 $08 N-06 E-1046 C$ $O B N-0 O E-141) C C 1$
CUTNER,M.A. MCCRAW

PHILLIPS,V.H.

JACKSON, G.

RARGER, W.H.

MUKGAN, C .

KONAWA CITY OK

KUNANA CITY UK

KUNAWA CITY UK

WOUOY, R.

DUOLEN, R

VAMOUSA SCHOOL

MULLINS,L.

RAPER,O

HALL,R.L.

CLARK, G. N.

SIAUFFER, C.

MULLEN,J.

HIOLLISIIN, 1 .

MAPCU INC

HEARI, J.T.

WIECK,J

UNKNUWN

UNKNUWN

NALLS, H.

BUWLEGS OK.

PARSIJH,J.

UNKAI)WN

HEISKIILL, J.J. 1963

SCHAEFFR,H. 1930
1970

\begin{tabular}{|c|c|c|}
\hline 1939 & 700 & 0 \\
\hline & 202 & 0 \\
\hline 1962 & 236 & 0 \\
\hline 1974 & 150 & 0 \\
\hline & 123 & 0 \\
\hline 1950 & 160 & 6 \\
\hline & 70 & 0 \\
\hline & 200 & 6 \\
\hline & 196 & 0 \\
\hline & 42 & 0 \\
\hline 1962 & 214 & 6 \\
\hline & 81 & 0 \\
\hline & 100 & 6 \\
\hline 1959 & 65 & 6 \\
\hline 1964 & 180 & 6 \\
\hline & 40 & 0 \\
\hline & 82 & 0 \\
\hline 1970 & 214 & 6 \\
\hline & 82 & 0 \\
\hline 1954 & 321 & 0 \\
\hline 1937 & 126 & 6 \\
\hline 190? & 217 & 6 \\
\hline 1969 & 180 & 6 \\
\hline & 143 & 0 \\
\hline & 125 & 0 \\
\hline & 75 & 0 \\
\hline & 92 & 6 \\
\hline & 131 & 0 \\
\hline 1972 & 550 & 6 \\
\hline & 59 & 0 \\
\hline 1470 & 721 & 5 \\
\hline & 410 & 0 \\
\hline 1963 & 176 & 6 \\
\hline 1930 & 500 & 0 \\
\hline
\end{tabular}

(FI) (MU-YR) (GAL/MIN)

(FT)

\begin{tabular}{|c|c|c|c|c|c|c|}
\hline B & 50 & A & $11=74$ & & $D E$ & 1042 \\
\hline \multirow[t]{2}{*}{6} & 37 & $A$ & $11-74$ & & $D E$ & 920 \\
\hline & 170 & A & $11-74$ & & DE & 950 \\
\hline 6 & 84 & $A$ & $11-74$ & & $D E$ & 855 \\
\hline \multirow[t]{5}{*}{6} & 24 & A & $5-70$ & & DA & 915 \\
\hline & 71 & A & & & DE & 920 \\
\hline & 39 & A & $11-74$ & & $D E$ & 902 \\
\hline & 61 & $A$ & $11-74$ & & $D E$ & 990 \\
\hline & 29 & A & $11-74$ & & $D E$ & 940 \\
\hline 6 & 36 & A & $11-74$ & & $D E$ & 965 \\
\hline 8 & 24 & A & $11-74$ & & $D E$ & 930 \\
\hline \multirow[t]{3}{*}{5} & 19 & A & $5-70$ & & UB & \\
\hline & 39 & A & $11-74$ & & $D E$ & 870 \\
\hline & & & & & $D E$ & 955 \\
\hline 6 & 36 & A & $11-74$ & & $D E$ & 950 \\
\hline \multirow[t]{2}{*}{6} & 41 & A & $11-74$ & . & $D E$ & 910 \\
\hline & & & & & $D E$ & 910 \\
\hline 6 & 49 & $A$ & $11-74$ & 3 & $D E$ & 865 \\
\hline \multirow[t]{2}{*}{6} & & & & & $D E$ & 930 \\
\hline & 15 & A & $11-74$ & & $D E$ & 915 \\
\hline 6 & 7 & A & $11-74$ & & $O E$ & 925 \\
\hline \multirow[t]{2}{*}{4} & 36 & A & $11-74$ & & DE & 920 \\
\hline & 117 & A & $11-21$ & & $D E$ & 915 \\
\hline 6 & 54 & $A$ & $11-74$ & & $D E$ & 900 \\
\hline 6 & 121 & A & $11-74$ & & $D E$ & 855 \\
\hline 6 & 16 & A & $11-74$ & & $D E$ & 910 \\
\hline 6 & 60 & A & $11-74$ & & DE. & 945 \\
\hline 6 & 87 & A & $11-74$ & & $D E$ & 870 \\
\hline \multirow[t]{2}{*}{6} & 46 & A & $11-74$ & & DE & 980 \\
\hline & 37 & A & $11-74$ & & $D E$ & 943 \\
\hline \multirow[t]{2}{*}{6} & 59 & A & $12-74$ & & $D E$ & 975 \\
\hline & 8 & $A$ & $11-74$ & & UE & 930 \\
\hline 6 & 32 & A & $11-74$ & & DE. & 925 \\
\hline \multirow[t]{4}{*}{6} & 23 & A & $11-74$ & & $D E$ & 925 \\
\hline & 284 & $A$ & $11-74$ & 145 & $D E$ & 970 \\
\hline & 50 & A & $5-70$ & & DA & 455 \\
\hline & 194 & A & $11-74$ & & $D t$ & 1020 \\
\hline \multirow[t]{2}{*}{6} & 184 & A & $11-74$ & & $O E$ & 875 \\
\hline & & & & & $D E$ & 920 \\
\hline 6 & 162 & A & $11-74$ & & DE & 955 \\
\hline
\end{tabular}


SEMINULE CIJUNTY

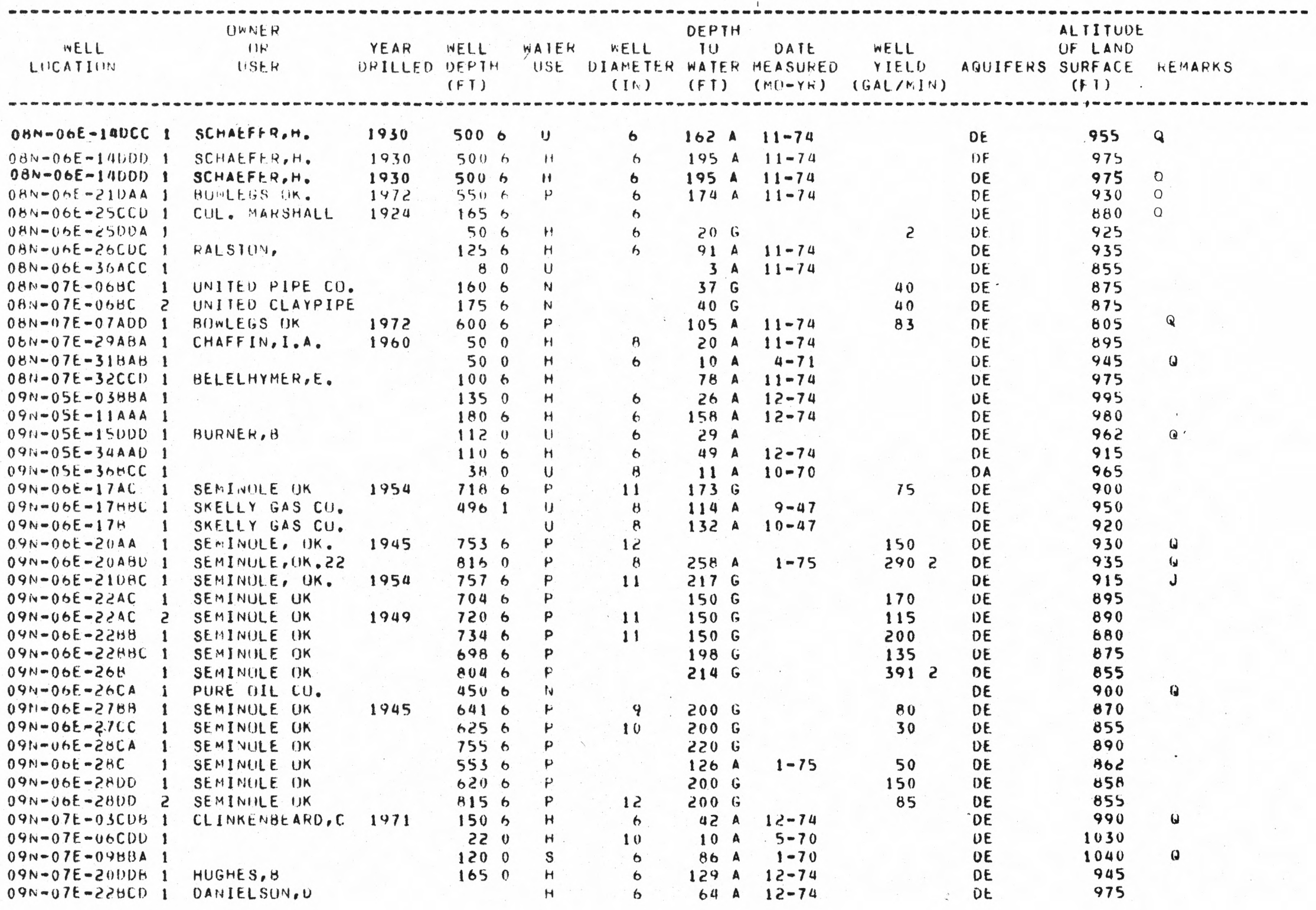


Table 1.--Records of selected wells.--Continued

SEMINULE CUUNITY

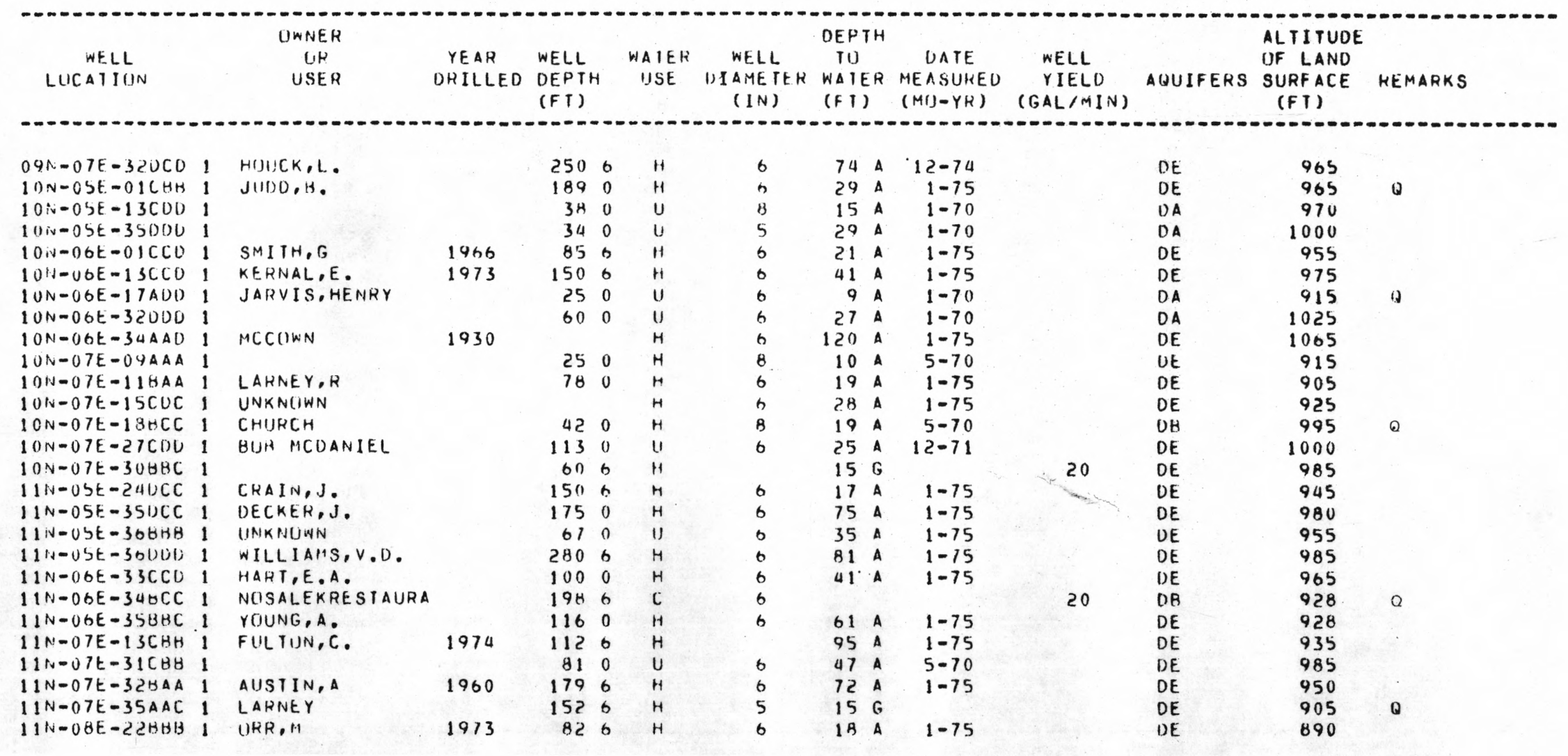


Table 1.--Records of selected wells.--Continued

PUTTANATUMIE CLUUNTY

\begin{tabular}{|c|c|c|c|c|c|c|c|c|c|c|c|c|c|c|}
\hline $\begin{array}{l}\text { WELL } \\
\text { LUCATIUN }\end{array}$ & & $\begin{array}{c}\text { UWNER } \\
\text { UIR } \\
\text { USER }\end{array}$ & $\begin{array}{c}\text { YEAR } \\
\text { DHILLED }\end{array}$ & $\begin{array}{l}\text { WELL } \\
\text { DEPIH } \\
(F T)\end{array}$ & & $\begin{array}{l}\text { WAIER } \\
\text { USE }\end{array}$ & $\begin{array}{l}\text { WELL } \\
\text { OI AMEIER } \\
\text { (IN) }\end{array}$ & $\begin{array}{l}\text { DEPI } \\
\text { TO } \\
\text { WATE } \\
\text { (FT) }\end{array}$ & & $\begin{array}{l}\text { DATE } \\
\text { MEASURED } \\
\text { (MU-YR) }\end{array}$ & $\begin{array}{c}\text { WELL } \\
\text { YIELU } \\
\text { (GAL/MIN) }\end{array}$ & ANUIFERS & $\begin{array}{l}\text { AL TITUUE } \\
\text { UF LAND } \\
\text { SUKFACE } \\
\text { (FT) }\end{array}$ & KEMARKS \\
\hline $08 N=05 E-33 A A K$ & 1 & MAUD,UK. & & 668 & 0 & $p$ & & & & & & DE & 945 & 0 \\
\hline $\begin{array}{l}08 N-05 E=33 A B B \\
08 N=05 E=33 A C C\end{array}$ & 1 & MAUL, IIK. & 1927 & $\begin{array}{l}650 \\
650\end{array}$ & 6 & $\begin{array}{l}s \\
p\end{array}$ & 6 & 238 & $A$ & $11-74$ & 25 & $D E$ & 950 & \\
\hline $\begin{array}{l}08 N=05 E=33 A C C \\
09 N=05 E-04 D C C\end{array}$ & $\begin{array}{l}1 \\
1\end{array}$ & $\begin{array}{l}\text { MAUU, UK, } \\
\text { JONES, IF }\end{array}$ & & $\begin{array}{l}650 \\
248\end{array}$ & $\begin{array}{l}6 \\
0\end{array}$ & $\begin{array}{l}P \\
H\end{array}$ & 6 & $\begin{array}{l}208 \\
140\end{array}$ & $A$ & $\begin{array}{r}11=74 \\
1-75\end{array}$ & 55 & $\begin{array}{l}D E \\
D E\end{array}$ & $\begin{array}{r}930 \\
1065\end{array}$ & o \\
\hline $09 N=05 E-08 H A A$ & 1 & EARL SHURU,IJK. & & 165 & 0 & U & 6 & 63 & A & $1-75$ & & $\mathrm{DE}$ & 1015 & \\
\hline (1) $9 N-05 E-08 C A A$ & 1 & EARLSBORIJ OK & & 700 & 6 & $u$ & 6 & 215 & $A$ & $1-75$ & & DE & 1015 & \\
\hline $09 N-05 E-17 C C U$ & 1 & UNKNOWN & & 53 & 0 & $u$ & & 15 & $A$ & $1-75$ & & DE & 955 & \\
\hline $09 N-05 E-29 A A A$ & $i$ & GWARINEY,J. & & 47 & 6 & $H$ & & 3 & A & & & $D E$ & 970 & \\
\hline $11 \mathrm{~N}-06 \mathrm{E}-06 \mathrm{CCC}$ & 1 & UIXUIN, J.M. & & 220 & 6 & $H$ & & 40 & G & & & DA & 983 & 0 \\
\hline $11 N=06 E-15 A D D$ & 1 & & & 72 & 0 & $u$ & 8 & 53 & $A$ & $6-70$ & & DB & 994 & \\
\hline $11 N-06 E-200$ & 1 & AFFENTROUGER, & v & 37 & 6 & $I$ & 6 & 9 & G & & 100 & OA & 885 & \\
\hline $11 N-06 E-27 A C$ & $i$ & WHI IE, MINNIE & & 45 & 6 & 1 & 36 & 20 & G & & 365 & OA & 895 & \\
\hline $11 N-06 E-27 A C$ & 1 & WHITE, MINNIE & & 43 & 6 & 1 & 36 & 20 & G & & 335 & $O A$ & 890 & \\
\hline
\end{tabular}


Table 1.--Records of selected wells.--Continued

GIKFUSKEE CUIINTY

\begin{tabular}{|c|c|c|c|c|c|c|c|c|c|c|c|}
\hline & OWNER & & & & & DEPTH & & & & ALTITUDE & \\
\hline $\begin{array}{c}\text { WELL } \\
\text { LUCATIUN }\end{array}$ & $\begin{array}{l}\text { IIR } \\
\text { USER }\end{array}$ & $\begin{array}{l}\text { YEAR } \\
\text { ORILLED }\end{array}$ & $\begin{array}{l}\text { WELL } \\
\text { DEPTH } \\
\text { (FI) }\end{array}$ & $\begin{array}{l}\text { WATER } \\
\text { USE }\end{array}$ & $\begin{array}{l}\text { WELL } \\
\text { DIAME TER } \\
\text { (IN) }\end{array}$ & $\begin{array}{l}\text { TO } \\
\text { WATER } \\
\text { (FT) }\end{array}$ & $\begin{array}{l}\text { DATE } \\
\text { MEASUREO } \\
\text { (MUII=YR) }\end{array}$ & $\begin{array}{c}\text { WELL } \\
\text { YIELD } \\
\text { (GAL/MIN) }\end{array}$ & AQUIFERS & $\begin{array}{l}\text { OF LAND } \\
\text { SURFACE } \\
\text { (FT) }\end{array}$ & REMARKS \\
\hline
\end{tabular}

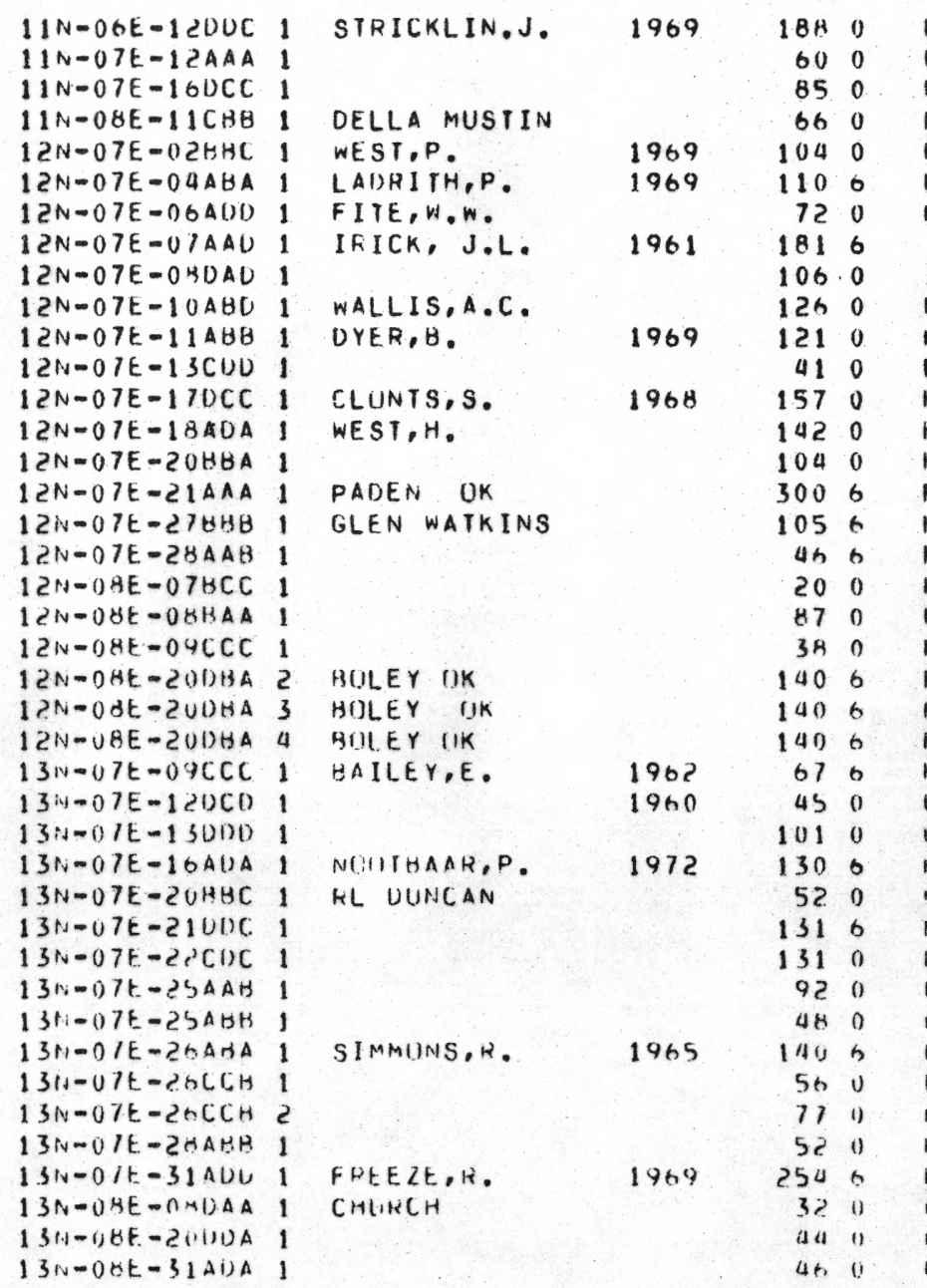

975
975
895
1015
0925
660
940
940
890
895
880
870
870
990
990
940
1010
965
915
918
910
950
900
900
800
845
818
850
800
900
890
835
880
830
840
832
930
890
850
850
925
7
90

0 
Table 1.--Records of selected wells.--Continued

GKF USKEE COUNTY

\begin{tabular}{|c|c|c|c|c|c|c|c|c|c|c|c|}
\hline & UNNER & & & & & DEPTH & & & & ALTITUDE & \\
\hline $\begin{array}{c}\text { NELL } \\
\text { LOCATIUN }\end{array}$ & $\begin{array}{c}\text { OR } \\
\text { USER }\end{array}$ & $\begin{array}{l}\text { YEAH } \\
\text { DRILLED }\end{array}$ & $\begin{array}{l}\text { WELL } \\
\text { DEPTH } \\
\text { (FI) }\end{array}$ & $\begin{array}{l}\text { WAIEH } \\
\text { USE }\end{array}$ & $\begin{array}{l}\text { WELL } \\
\text { DIAMETER } \\
\text { (IN) }\end{array}$ & $\begin{array}{l}\text { TO } \\
\text { WATER } \\
\text { (FT) }\end{array}$ & $\begin{array}{l}\text { DATE } \\
\text { MEASURED } \\
\text { (MU-YR) }\end{array}$ & $\begin{array}{c}\text { WELL } \\
\text { YIELU } \\
\text { (GAL/MIN) }\end{array}$ & AGUIFERS & $\begin{array}{l}\text { OF LAND } \\
\text { SURFACE } \\
\text { (FI) }\end{array}$ & REMARKS \\
\hline
\end{tabular}

$13 N-08 E-32 C D O 1$

1974

$5000 \quad H$

6

32 10-74

47 A $10-74$

DE
DE

885

$13 N-0 B E-3200 C$

$540 \quad U$

DE

910 
LINCOLN COUNTY

\begin{tabular}{|c|c|c|c|c|c|c|c|c|c|c|c|}
\hline & UWNER & & & & & DEPTH & & & & ALTITUOE & \\
\hline WELL & IIR & YEAR & WELL & WAIER & WELL & Tu & DATE & WELL & & UF LAND & \\
\hline LUCA IION & USER & DRILLED & $\begin{array}{l}\text { DEPTH } \\
\text { (FT) }\end{array}$ & USE & $\begin{array}{c}\text { OIAME TEK } \\
\text { (IN) }\end{array}$ & $\begin{array}{l}\text { WATEH } \\
\text { (FI) }\end{array}$ & $\begin{array}{l}\text { MEASUREU } \\
(M()-Y R)\end{array}$ & $\begin{array}{c}\text { YIELD } \\
(G A L / M I N)\end{array}$ & AQUIFERS & $\begin{array}{l}\text { SURF ACE } \\
\text { (FT) }\end{array}$ & KEMARKS \\
\hline
\end{tabular}

$1211-05 E-35100 \mathrm{C} \quad 1$

$12 N .0 O E-01 A A A$

$12 N-06 E-06 B A H$

$12 N=06 E-10 B C C$

$12 N-06 E-20 D A C$

$12 N=06 E-201 D D H$

$12 N-0 D E-22 B C B$

$12 N-00 t-24 C C C 1$

$12 N-0 B E-2 R U A D$ $12 N-06 E-24 C C A$

$13 N-06 E-0 O A A A 1$

N $13 N-00 E-14001$

$1314-06 E-110002$

$13 N-06 E-25 B C C$

$13 N-06 E-2800 C$

$13 N=06 E-29 A A A$,

$13 N-06 E-31 A D O 1$

$14 N-06 E-02 A A D$

$14 N-0 B E-11 A B H$

$14 N=00 E-35 B C C \quad 1$

$15 \mathrm{~N}=06 \mathrm{OCE}=02 \mathrm{CCH} 1$

$15 N-00 E-078 A B$.

$15 N-0 h E-10 E B A 1$

$15 N-00 E-12 B C H$

$15 N-06 E-12 C B H$

$15 N-00 E-15 E 001$

$15 N-06 E-1700 B$

$15 N-00 E-2 B A A A$

$15 \mathrm{~N}-06 \mathrm{E}-2 \mathrm{CUAD}$

$15 N-06 E-2 A D H O$

$15 N-0 O E-3 S B A A$

$16 N-1) 5 E-23000$

$16 N-05 E-36 B B C .1$

$16 N-06 E-030 C D$

$16 N-U 6 E-O S C C C$

$16 N-06 E-22 A B H$

$16 N-V O E-23 B B B$

$16 N-0 B E-33 A C D$

$17 N-05 E-23 B$
WHITE, D.

JAMES,H.

PRAGUE WELL 7

PRAGIJE WELL 5

PRAGUE WELL 1

PRAGUE, OK

PRAGUE WELL 8

OSUSKY,F.

MAYES, W

PRUITT,J.

DAVIS,LLUYO

LONERY,D.

MCELYEA,B

CHANCES R FARM

MURPHY, S

KIDUS, H.

STRUUU, 1.JK.

BUNYARU,

STROUD, OK 12

STRIJUD UK $5 \quad 195$

STRIJUU OK $11 \quad 1966$

1958

IRAWICK RANCH

REMINGTON

HARRISIJN

SILL,J,L

SINCLAIR PIPE

\section{0}

1736

$\begin{array}{ll}71 & 0\end{array}$

670

367 h

3566

4206

1240.

412

3746

460

46

$\begin{array}{ll}77 & 0 \\ 74 & 0\end{array}$

740
1376

1830

1260

1006

1806

690

620

77

1906

1240

1300

1260

$260 \mathrm{~h}$

1206

410

3390

2650

4083

2486

1966

1150

430

1111

1071

976

1606

3576

$\mathrm{U}$
$H$
$\mathrm{U}$
$\mathrm{P}$
$\mathrm{P}$
$\mathrm{P}$
$H$
$\mathrm{P}$
$\mathrm{P}$
$\mathrm{H}$
$\mathrm{U}$
$\mathrm{H}$
$\mathrm{H}$
$\mathrm{H}$
$\mathrm{H}$
$\mathrm{H}$
$\mathrm{U}$
$\mathrm{U}$
$\mathrm{H}$
$\mathrm{H}$
$\mathrm{H}$
$\mathrm{U}$
$\mathrm{U}$
$\mathrm{H}$
$\mathrm{U}$
$\mathrm{H}$
$\mathrm{P}$
$\mathrm{P}$
$\mathrm{H}$
$\mathrm{S}$
$\mathrm{U}$
$\mathrm{U}$
$H$
$H$
$H$
$\mathrm{H}$

$\begin{array}{rlr}64 & A & 1-75 \\ 72 & A & 10-74 \\ 17 & A & 1-75 \\ 21 & A & 1-75 \\ & & 5-75 \\ 172 & A & 5-75 \\ 56 & A & 1-75 \\ 166 & G & 5-29 \\ & & 5-75 \\ 7 & A & 1-75 \\ 18 & A & 10-74 \\ 17 & A & 1-75 \\ 63 & A & 10-74 \\ 40 & A & 1-75 \\ 12 & A & 1-75 \\ 27 & A & 1-75 \\ 128 & A & 10-74 \\ 64 & A & 2-75 \\ 32 & A & 2-75 \\ 37 & A & 10-74 \\ 41 & A & 10-74 \\ 44 & A & 10-74 \\ 67 & A & 10-74 \\ 81 & A & 10-74 \\ 30 & A & 10-74 \\ 53 & A & 10-74 \\ 22 & A & 10-74 \\ 182 & A & 6-75 \\ 60 & A & 6-75 \\ 130 & A & 7-75 \\ 83 & A & 10-74 \\ 25 & A & 2-75 \\ 29 & A & 2-75 \\ 7 & A & 2-75 \\ 32 & A & 2-75 \\ 76 & A & 3-75 \\ 21 & 6 & 3-75 \\ 91 & A & 10-74 \\ 85 & A & 8-52 \\ & & \end{array}$

1005

890

850

780

25

1010

970

1005

850

860

860

860

825

910

910

885

845

810

922

960

905

910

890

925

885
825

890

880

900

960

935

1005

910

1025

990

945

980
O 
Table 1.--Records' of selected wells.--Continued

CREEK CUUNTY

\begin{tabular}{|c|c|c|c|c|c|c|c|c|c|c|c|}
\hline & OWNER & & & & & DEPIH & & & & AL II ITUDE & \\
\hline WELL & OR & YEAR & WELL & WAIEK & WELL & TU & DATE & WELL & & OF LAND & \\
\hline LUCATIIIN & USER & URILLED & $\begin{array}{l}\text { DEPIH } \\
\text { (FT) }\end{array}$ & USE. & $\begin{array}{c}\text { OIAME IER } \\
\text { (IN) }\end{array}$ & $\begin{array}{l}\text { WAIER } \\
\text { (FI) }\end{array}$ & $\begin{array}{l}\text { MEASUKED } \\
(M U-Y R)\end{array}$ & $\begin{array}{c}\text { YIELD } \\
\text { (GAL/MIN) }\end{array}$ & AQUIFERS & $\begin{array}{l}\text { SURF ACE } \\
(F I)\end{array}$ & REMARKS \\
\hline
\end{tabular}

IUN-OTE-OSCUC I MCCAMMUN OHOBO

$14 N-07 E-0 Y C C B$

$14 N-07 E=09 A B A 1$

$14 N-07 E-10 C B B 1$

$14 N-07 E-12 C C C 1$

WALKER, B.

HOIIO

GOOUE, E.

$-\infty+\infty-\infty$

(FI) (MU-YR)

(GAL/MIN)

(FT)

$15 N-07 E-10 C B I)$

$1 S N-07 E-13 B A A$

$15 N-07 E-24 A A D 1$

$15 N-07 E-25 A A D$

$15 N-07 E-25 C C C$

MCGUIRE,E.

$\begin{array}{rlr}57 & A & 10-74 \\ 89 & A & 9-70 \\ 146 & A & 10-74 \\ 113 & G & \\ 37 & A & 10-74 \\ 20 & G & \\ 24 & A & 10-70 \\ 77 & A & 10-74 \\ 90 & G & 10-74 \\ 87 & A & 10-74 \\ 29 & A & 10-74 \\ 65 & A & 10-74\end{array}$

$15 N-07 E-27 B B B \quad 1$

$15 N-07 E-27 B H C 1$

$15 N-07 E-30 D A B \quad 1$

$15 N-07 E-32 H B C 1$

$15 N-0 B E-013 B B C$

$15 N-09 E-06 B$

GAITHER, 8.

HAYHURST, O.B.

DEPEW UKLA

(10 0

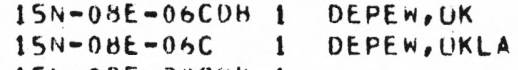

$15 N-0 B E-20(01) 1$

$15 N-0 B E-29 H C B \quad 1$

$15 N-08 E-30 C C B \quad 1$

$16 N-07 t-09 B A$

$16 N-07 E-110 C C 1$

$16 N-07 E-110 \mathrm{CC} 2$

$16 N-07 E-14 C A C$ I

$16 N-07 E-140 B C 1$

$1 B N-07 E-1 B A K B \quad 1$

16N-07E-21ADA 1

$16 N-07 E-3300 A$

$16 N-07 E-35 A B$

$16 N-08 E-22 C C D 1$

$1 I N-06 E-01 A A A 1$

$17 N-06 E-34 B 8 B$

$17 N-07 E-06 A C B 1$

$17 N-07 t-08 C B C 1$

$17 N-07 E-0 B C B U 1$

$17 N-07 E-08 C C A$

HALSTEAD, C.

GULF UIL CO.

1973

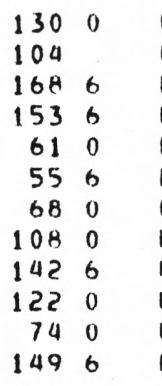

$956 \quad \mathrm{H}$

$\begin{array}{lll}58 & 0 & U\end{array}$

$9500 \quad 4$

$2160 \mathrm{~S}$

1626

2006

1246

1856

$710 . \mathrm{H}$

$2 \mathrm{H} O \mathrm{U}$

107

6506

$460 \quad U$

$450 \mathrm{H}$

$300 \mathrm{H}$

$40 \mathrm{O}$

$290 \quad H$

COBGLE,ALVIN $1967 \quad 117 \mathrm{~b} \quad \mathrm{H}$

UALEY R HIIPPES

996

6506

DRUMRIGHT, UK

HAPTIST CHURCH

DRUMRIGHT, UK

DRUMRIGHT,UK

DRUMRIGHI UK.

1930

5006

$26060 \%$

$4036 \quad P$

$425 \cup \quad h$

$4876 \quad P$

$17 N-07 E-2 S A O D \quad 1$

DRUMRIGHI,UK

$$
\begin{array}{rrr}
35 & G & - \\
20 & A & 11-70 \\
36 & A & 10-74 \\
108 & A & 10-74
\end{array}
$$

$3 \mathrm{G}$

10074

25 10-74

$22 \quad 10-70$

72 10-74

$206 \mathrm{G}$

35 A 11-70

28 A $2-75$

$\begin{array}{ll}21 & A 1-70 \\ 26 & 11-70\end{array}$

2ट A $2-75$

12
8

776

65 A

$98 \mathrm{G}$

$85 \mathrm{G}$

$75 \mathrm{G}$

13219

$135 \mathrm{G}$

$40 \mathrm{G}$

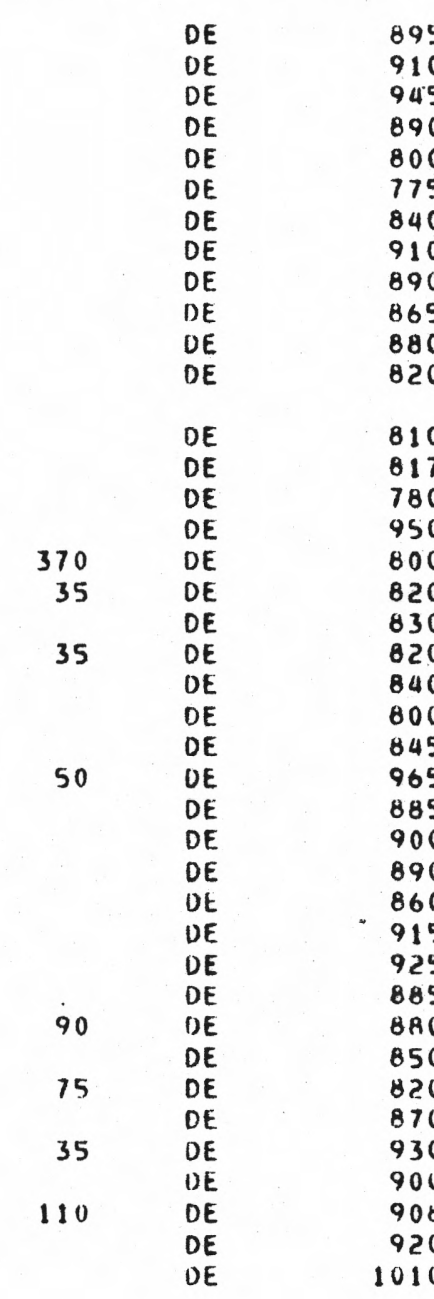

Q

$\theta$

840

890

865

880

$820 \quad 0$

810

817

780
950

800

820

830
820

840

845

885

900

890

860

925

885

$8 R O$

820

870

930

906

920
010 
Table 1.--Records of selected wells.--Continued

CREEK COUNIY

\begin{tabular}{|c|c|c|c|c|c|c|c|c|c|c|c|}
\hline & OWNER & & & & & DEPTH & & & & ALTITUDE & \\
\hline $\begin{array}{c}\text { WEI.L } \\
\text { LUCATIUN }\end{array}$ & $\begin{array}{c}\text { OR } \\
\text { USER }\end{array}$ & $\begin{array}{l}\text { YEAR } \\
\text { DRILLED }\end{array}$ & $\begin{array}{l}\text { WELL } \\
\text { DEPTH } \\
\text { (FI) }\end{array}$ & $\begin{array}{l}\text { WATER } \\
\text { USE }\end{array}$ & $\begin{array}{l}\text { NELL } \\
\text { OIAMEIER } \\
\text { (IN) }\end{array}$ & $\begin{array}{l}\text { IU } \\
\text { WATEK } \\
\text { (FT) }\end{array}$ & $\begin{array}{l}\text { DATE } \\
\text { MEASURED } \\
\text { (MO-YR) }\end{array}$ & $\begin{array}{c}\text { WELL } \\
\text { YIELD } \\
\text { (GAL/MIN) }\end{array}$ & AQUIFEKS & $\begin{array}{l}\text { OF LAND } \\
\text { SURFACE } \\
\text { (FI) }\end{array}$ & REMARKS \\
\hline
\end{tabular}

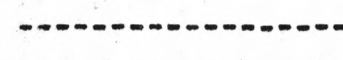

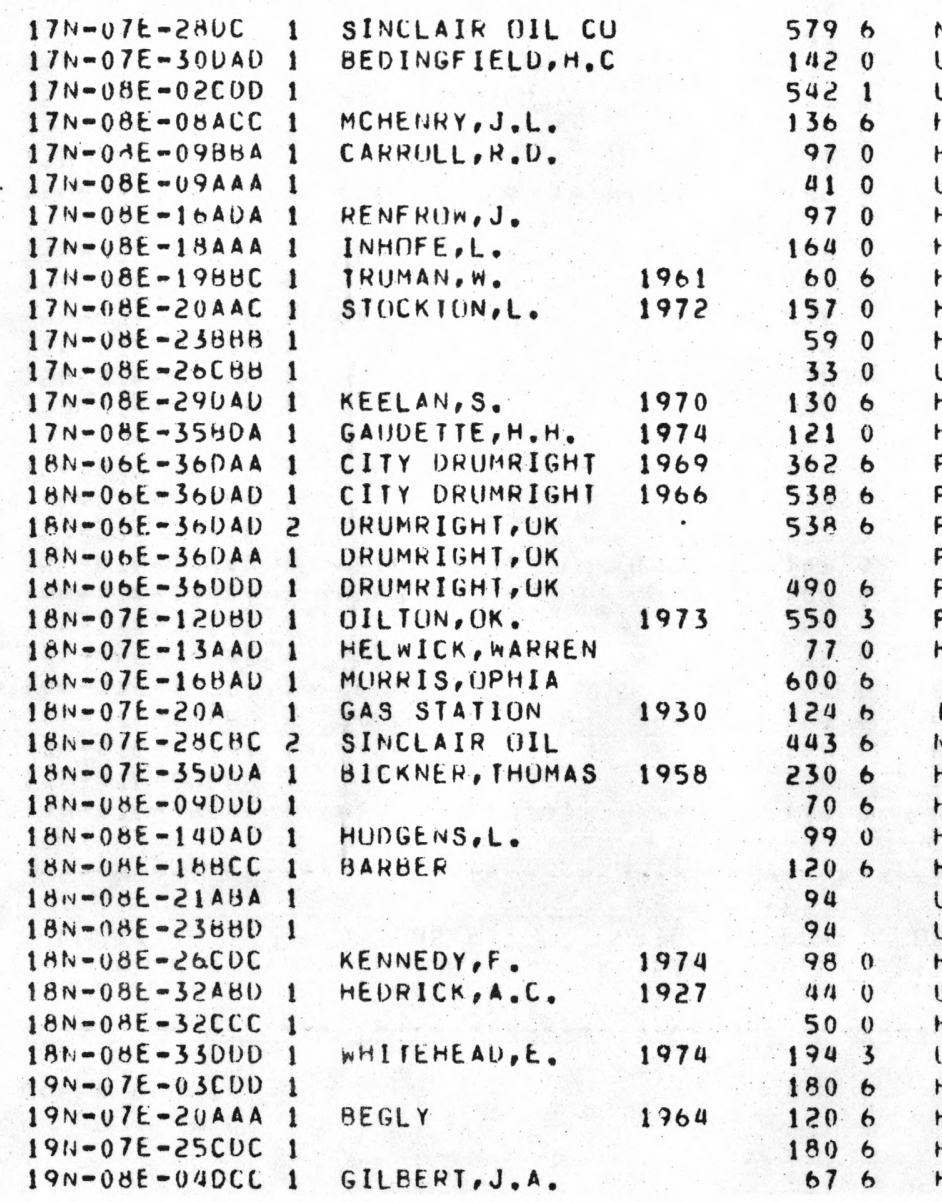

1
$(1 N)$

\begin{tabular}{|c|c|c|c|}
\hline & 275 & G & \multirow[b]{2}{*}{$1-75$} \\
\hline 6 & 60 & A & \\
\hline \multirow[t]{2}{*}{8} & 21 & A & $12-74$ \\
\hline & 34 & A & $10-74$ \\
\hline \multirow[t]{2}{*}{6} & 25 & A & $12-74$ \\
\hline & 22 & A & $10-74$ \\
\hline \multirow[t]{4}{*}{6} & 44 & A & $10-74$ \\
\hline & 30 & $A$ & $10-74$ \\
\hline & 119 & A & $10-74$ \\
\hline & 20 & A & $10-74$ \\
\hline \multirow[t]{2}{*}{4} & 21 & $A$ & $1-75$ \\
\hline & 33 & $A$ & $10-74$ \\
\hline \multirow[t]{2}{*}{6} & 24 & $A$ & $1-75$ \\
\hline & 87 & $A$ & $10-75$ \\
\hline \multirow[t]{3}{*}{8} & 206 & A & $8-75$ \\
\hline & 175 & G & \\
\hline & 100 & G & \\
\hline \multirow{3}{*}{$\begin{array}{l}8 \\
6\end{array}$} & & . & \\
\hline & 64 & A & $8-71$ \\
\hline & 276 & A & $4-75$ \\
\hline 11 & 265 & $\mathrm{G}$ & $9-70$ \\
\hline 6 & 45 & G & $4-75$ \\
\hline \multirow[t]{2}{*}{6} & 42 & A & $2-75$ \\
\hline & 50 & fo & $11-72$ \\
\hline 6 & 73 & A & $12-74$ \\
\hline \multirow[t]{2}{*}{6} & 73 & $A$ & $12-74$ \\
\hline & 14 & A & $1-75$ \\
\hline s & 8 & A & $1-75$ \\
\hline 8 & 35 & A & $1-15$ \\
\hline 6 & 22 & A & $1-75$ \\
\hline 0 & 60 & G & $2-73$ \\
\hline 6 & 105 & G & $4-75$ \\
\hline b & & & \\
\hline 6 & 35 & G & $2-73$ \\
\hline
\end{tabular}

\begin{tabular}{|c|c|c|c|}
\hline \multirow[t]{13}{*}{20} & DE & 1040 & \\
\hline & $D E$ & 1030 & \\
\hline & $D E$ & 885 & \\
\hline & $D E$ & 920 & $\omega$ \\
\hline & DE & 910 & \\
\hline & $D E$ & 950 & \\
\hline & $D E$ & 850 & \\
\hline & $D E$ & 930 & \\
\hline & DE & 1010 & \\
\hline & DE & 945 & \\
\hline & $D E$ & 900 & \\
\hline & DE & 960 & \\
\hline & UE & 925 & \\
\hline \multirow[t]{3}{*}{30} & $D E$ & 960 & $\omega$ \\
\hline & DE & 960 & $R$ \\
\hline & $D E$ & 940 & $E_{Q}$ \\
\hline \multirow[t]{2}{*}{60} & $D E$ & 905 & \\
\hline & $D E$ & 900 & \\
\hline \multirow[t]{5}{*}{75} & $D E$ & 890 & . \\
\hline & UE & 861 & DE \\
\hline & $D E$ & 850 & $\mathrm{O}$ \\
\hline & $D E$ & 940 & $\mathrm{Q}$ \\
\hline & & 870 & 0 \\
\hline \multirow[t]{4}{*}{33} & $D E$ & 830 & \\
\hline & & 1000 & $\theta$ \\
\hline & $D E$ & 935 & \\
\hline & $D E$ & 990 & \\
\hline \multirow[t]{8}{*}{40} & $D E$ & 800 & $Q$ \\
\hline & $D E$ & 905 & \\
\hline & $D E$ & 905 & \\
\hline & $D E$ & 890 & \\
\hline & DE & 890 & \\
\hline & DE & 880 & i \\
\hline & $D E$ & 925 & 0 \\
\hline & $D E$ & 810 & $Q$ \\
\hline \multirow[t]{3}{*}{4} & vt & 860 & \\
\hline & DE & 823 & \\
\hline & OT & 800 & $Q$ \\
\hline
\end{tabular}


Table 1.--Records of selected wells.--Continued

PAYNE CIJUNIY

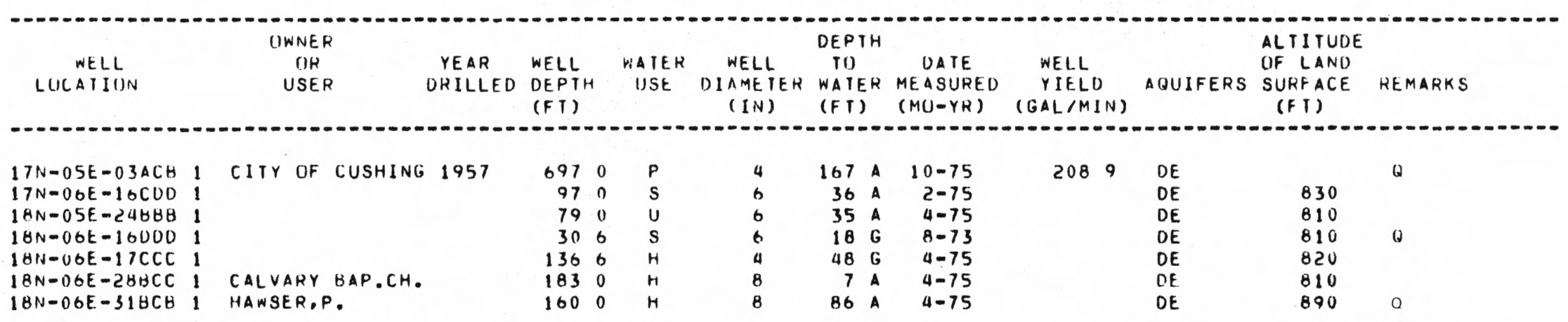


Table 1.--Records of selected wells.--Continued

PARNEE CUUNIY

\begin{tabular}{|c|c|c|c|c|c|c|c|c|c|c|c|}
\hline & UIWNER & & & & & DEPTH & & & & ALIITUDE & \\
\hline $\begin{array}{c}\text { WELL } \\
\text { LOCATIUN }\end{array}$ & $\begin{array}{l}\text { UIR } \\
\text { USER }\end{array}$ & $\begin{array}{l}\text { YEAR } \\
\text { ORILLED }\end{array}$ & $\begin{array}{l}\text { WELL } \\
\text { DEPIH } \\
\text { (FI) }\end{array}$ & $\begin{array}{l}\text { WAIER } \\
\text { USE }\end{array}$ & $\begin{array}{l}\text { VELL } \\
\text { DIAMETER } \\
\text { (IN) }\end{array}$ & $\begin{array}{l}\text { TU } \\
\text { WATER } \\
\text { (FT) }\end{array}$ & $\begin{array}{l}\text { UATE } \\
\text { MEASURED } \\
\text { (MU-YR) }\end{array}$ & $\begin{array}{c}\text { WELL } \\
\text { YIELI) } \\
\text { (GAL/MIN) }\end{array}$ & AGUIFERS & $\begin{array}{l}\text { OF LAND } \\
\text { SURFACE } \\
\text { (FT) }\end{array}$ & REMARKS \\
\hline
\end{tabular}

(IN)

(FT) (MU-YR) (GAL/MIN)

(FT)

$1 B N-0 B E-31 B C B \quad 1$

2ON-07E-01CDD

LONG OLI

2ON-07E-0ICDD

LONG OLIS W.

1961

1376

2OR-OTE-OSACA 1

$20 N-07 E-080 D D$

$20 N-07 E-27 B A O$

$2 O N-08 E-O S O U A 1$

$20 N-0 B E-O A C C C$

$2 O N-0 B E-U 9 D C D$

$20 N-08 E-12 C C C$

$2 O N-0 B E-17 A A D$

$2 O N-08 E-190 C A 1$

$20 N-08 E-190 C B$

$21 N-07 E-21 \triangle D B$

$21 N-07 E-24 C 00$

$21 N-07 E-24 C C O$

$21 \mathrm{~N}-07 \mathrm{E}-34 \mathrm{CCD}$

$21 \mathrm{~N}=0 \mathrm{BE}-200 \mathrm{CA}$

$21 N=08 E-29 A A 0$

$22 N-07 E-15000$

$22 N-07 E-2001 A$

$22 N-07 E-27 A B Q$
MCKENZIE THUMAS

LAMA WILKINS

DUNALO SIELING 1968

RWD 2 PAWNEE CO.

RWD ZPAWNEE CU.

DENPURT

1972

HC WALKER 1966

$\begin{array}{rrr}137 & 6 & H \\ 90 & 6 & H \\ 142 & 0 & U \\ 100 & & U \\ 200 & 0 & U \\ 28 & 6 & H \\ & & H \\ 20 & 6 & H \\ 114 & 6 & H \\ 80 & 6 & H \\ 128 & 6 & H \\ 124 & 6 & H \\ 266 & 6 & H \\ 90 & 1 & H \\ 156 & 6 & H \\ 35 & 0 & U \\ 85 & 6 & H \\ 65 & 6 & H \\ 160 & 6 & H \\ 92 & 0 & U\end{array}$

$\begin{array}{lll}60 \text { G } & 4-74 \\ 70 \text { A } & 5-72 \\ 192 \text { A } & 5-72 \\ 50 \text { G } & 4-75 \\ 60 \text { G } & 4-75 \\ 60 \text { G } & 4-75 \\ 44 \text { A } & 4-75 \\ 15 \text { A } & 5-72 \\ & & \\ 34 \text { A } & 5-72\end{array}$

Q

890
900

900
850
910

910

1005
915

990

790

860
825

825

860
960

960

890

905

905
930
800

920 
Table 1.--Records of selected wells.--Continued

OSAGE CUUNTY

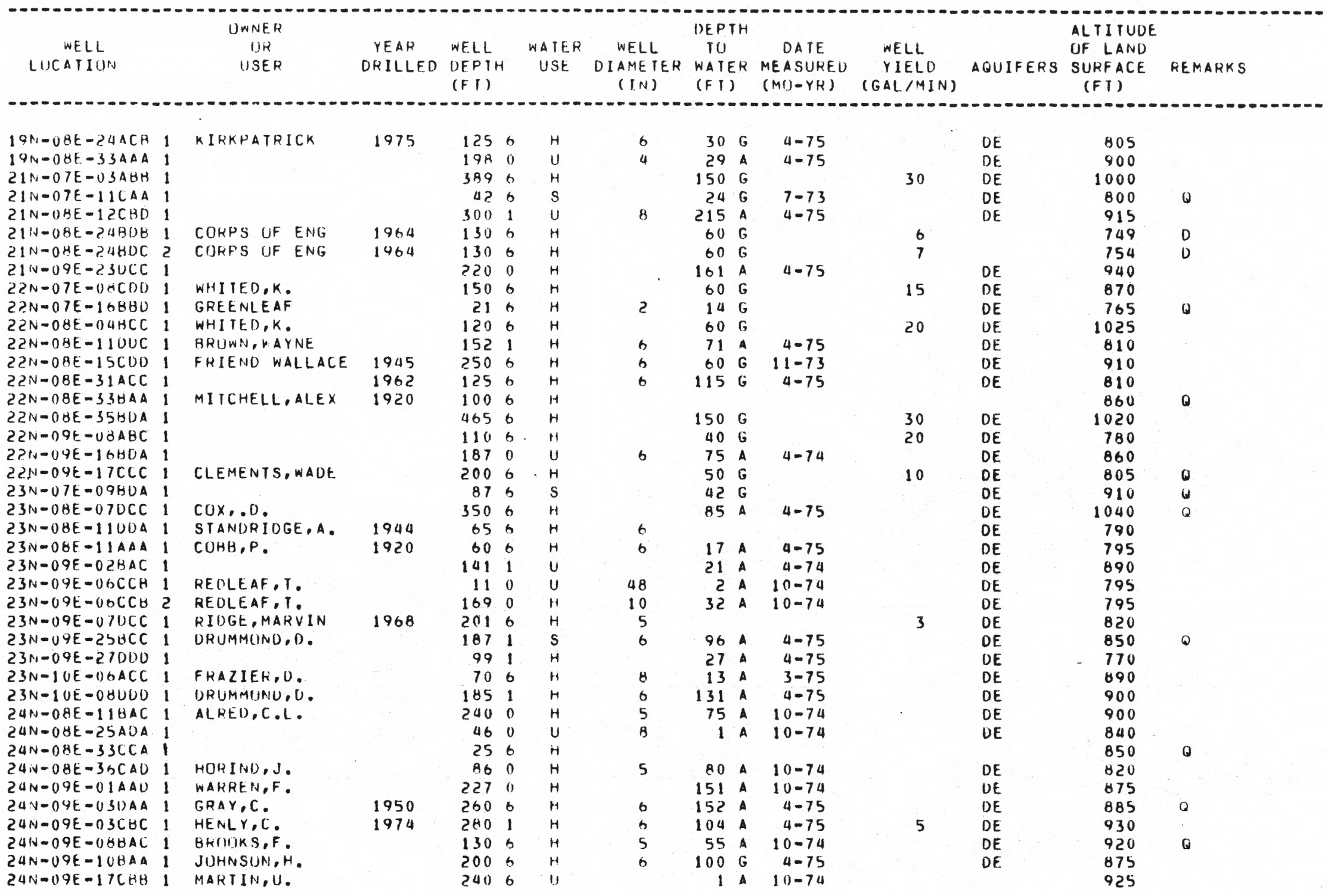


USAGE CHUNIY

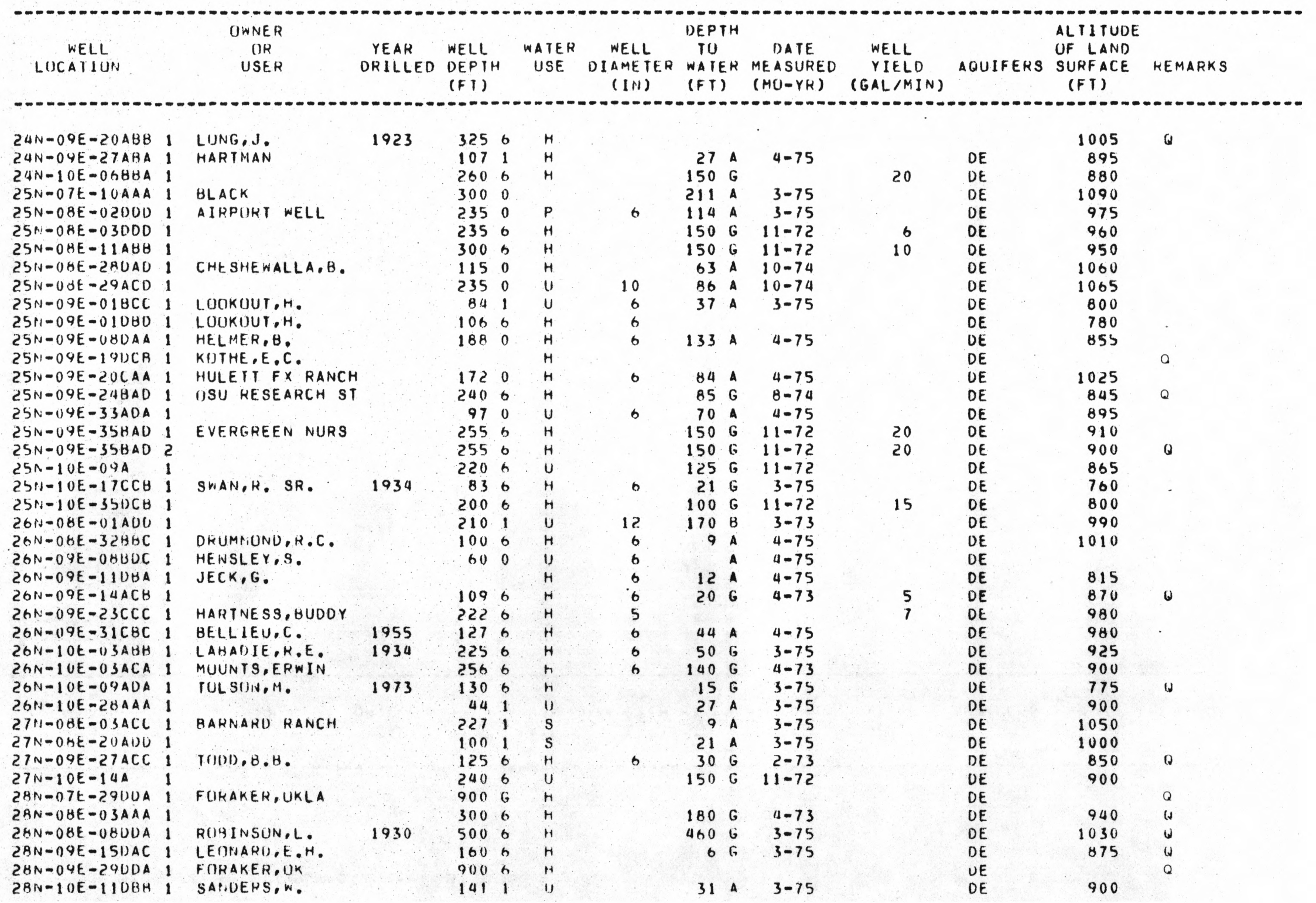


Table 1.--Records of selected wells.--Continued

OSAGE COUNTY

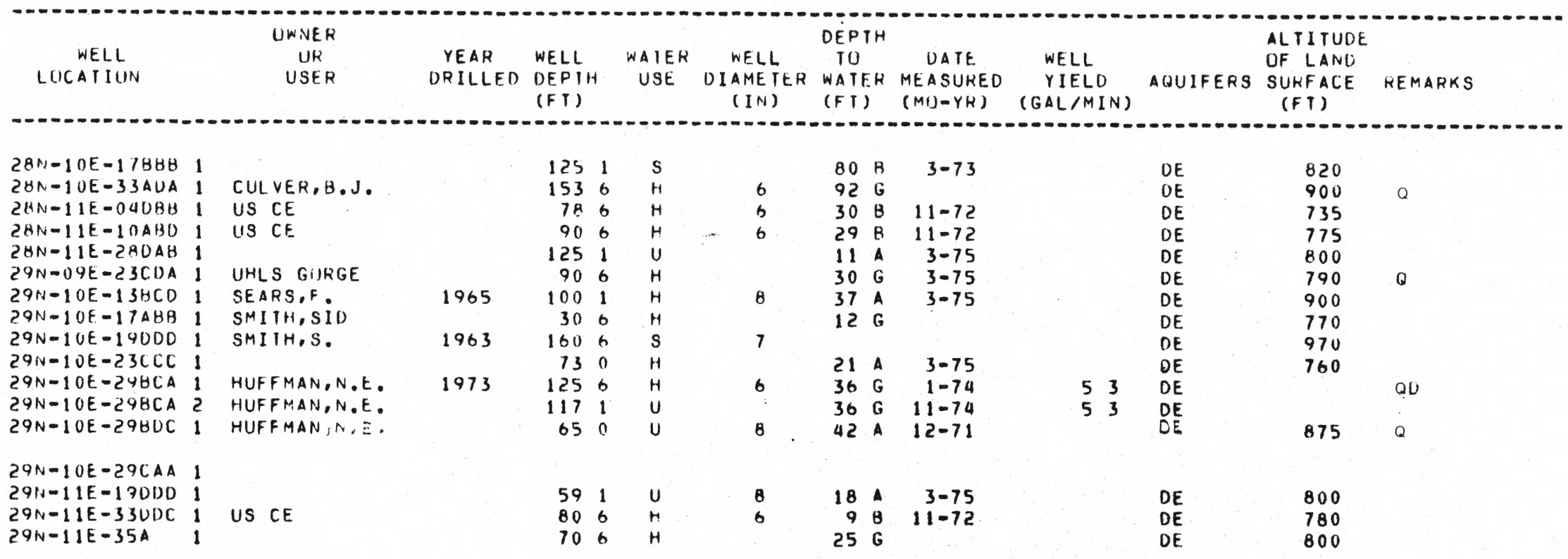


Table 2.--Chemical analyses of water from selected wells.

SEMINULE CUUNTY

\begin{tabular}{|c|c|c|c|c|c|c|c|c|c|c|c|c|c|c|c|c|}
\hline \multicolumn{4}{|c|}{$\begin{array}{c}\text { LUCAL WELL } \\
\text { NUMBER }\end{array}$} & $\begin{array}{r}\text { WELL } \\
\text { DEPTH }\end{array}$ & \multicolumn{3}{|c|}{$\begin{array}{c}\text { DATE } \\
\text { OF } \\
\text { COLLECIIUN }\end{array}$} & CALCIUM & MAGNESIUM & $\begin{array}{l}\text { SIJDIUM } \\
(N A+K)\end{array}$ & BICARBUINATE & SULFAIE & CHLURIDE & NITRATE & BRUMIDE & $\begin{array}{l}\text { DISSUL VED } \\
\text { SULIIIS }\end{array}$ \\
\hline & $0 S F$ & ZSUAD & -1 & 236 & 4 & 12 & 74 & 760 & & 180.0 & 426 & 160.0 & 64.0 & 19.0 & 0.20 & 788 \\
\hline $\begin{array}{l}O S N \\
\text { OSN }\end{array}$ & $\begin{array}{l}\text { OSE } \\
\text { OSE }\end{array}$ & $\begin{array}{l}\text { CODAO } \\
10000\end{array}$ & $\begin{array}{l}1 \\
1\end{array}$ & 230 & 11 & 13 & $\begin{array}{l}74 \\
74\end{array}$ & $\begin{array}{r}76.0 \\
4.8\end{array}$ & $\begin{array}{r}22.0 \\
4.2\end{array}$ & $\begin{array}{l}180.0 \\
150.0\end{array}$ & $\begin{array}{l}426 \\
336\end{array}$ & 27.0 & 15.0 & 0.4 & 0.10 & \\
\hline $07 \mathrm{~N}$ & $O 6 E$ & $23 B C B$ & 1 & 321 & 11 & 15 & 74 & 8.1 & 3.6 & 280.0 & 281 & 110.0 & 210.0 & & 0.70 & $7 R S$ \\
\hline $07 N$ & $06 \mathrm{E}$ & 33800 & 1 & 126 & 11 & 14 & 74 & 99.0 & 59.0 & 67.0 & 502 & 52.0 & 98.0 & 1.7 & 0.00 & 652 \\
\hline $07 \mathrm{~N}$ & $07 E$ & OSABB & 1 & 180 & 12 & 04 & 74 & 65.0 & 44.0 & 14.0 & 386 & 44.0 & 15.0 & & 0.1 & 387 \\
\hline $08 N$ & OOE & $10 \mathrm{BBC}$ & 1 & 178 & 03 & 10 & 64 & 12.0 & 35.0 & 390.0 & 452 & 215.0 & 320.0 & 1.0 & & 1300 \\
\hline $08 N$ & OOE & $14 D C C$ & 1 & 500 & 11 & 22 & 74 & 280.0 & 150.0 & 950.0 & 324 & 21.0 & 2300.1 & & 8.20 & 4500 \\
\hline $08 \mathrm{~N}$ & O6E & $1400 D$ & 1 & & 12 & 14 & 75 & 510.0 & 190.0 & 1900.0 & 306 & 21.0 & 4400.0 & & 2.60 & 7860 \\
\hline $08 \mathrm{~N}$ & OGE & 14000 & 1 & 500 & 11 & 22 & 74 & 2.9 & 1.7 & 160.0 & 349 & 29.0 & 14.0 & & 0.10 & \\
\hline$O 8 N$ & OOE & $14 D C C$ & 1 & & 12 & 15 & 75 & 3.6 & 2.0 & 160.0 & 346 & 32.0 & 16.0 & & 0.10 & 406 \\
\hline$O B N$ & OOE & 21UAA & 1 & 550 & 11 & 22 & 74 & 1.7 & 00.2 & 190.0 & 454 & 18.0 & 9.2 & 1.4 & 0.00 & 474 \\
\hline$O \mathrm{BN}$ & loE & $25 C C 0$ & 1 & 105 & 11 & 20 & 74 & 45.0 & 25.0 & 5.7 & 251 & 11.0 & 7.5 & & 0.10 & 220 \\
\hline $08 \mathrm{~N}$ & $07 E$ & OIAUD & 1 & 600 & 11 & 22 & 74 & 48.0 & 21.0 & 8.3 & 232 & 14.0 & 8.4 & & 0.00 & 221 \\
\hline $08 \mathrm{~N}$ & $07 E$ & $31 B A B$ & 1 & 50 & 04 & 26 & 71 & & & 20.0 & 186 & 21.0 & 8.0 & 0.3 & & 194 \\
\hline $09 \mathrm{~N}$ & $05 E$ & 15000 & 1 & 112 & 12 & 18 & 74 & & & & & & 62.0 & & 0.20 & 2020 \\
\hline $09 N$ & $O G E$ & $20 A A$ & 1 & 753 & 12 & 10 & 47 & 186.0 & 33.0 & 79.0 & 132 & 601.0 & 25.0 & 1.0 & & 108 \\
\hline $09 \mathrm{~N}$ & OOE & $20 A 80$ & 1 & 816 & 01 & 22 & 75 & 25.0 & 5.7 & BS. 0 & 208 & 48.0 & 6.5 & . & & 343 \\
\hline $09 N$ & UOE & $26 C A$ & 1 & 450 & 09 & 25 & 47 & 146.0 & 12.0 & 356.0 & 135 & 796.0 & 175.0 & & & 1540 \\
\hline $09 \mathrm{~N}$ & $07 E$ & $03 C D B$ & 1 & 150 & 12 & 17 & 74 & 11.0 & 4.6 & 5.4 & 51 & 6.2 & 5.7 & & & $7 b$ \\
\hline $09 N$ & $07 E$ & 09BBA & 1 & 120 & 05 & 22 & 70 & & & 8.0 & 142 & 11.0 & 9.1 & 83.0 & & 165 \\
\hline $10 N$ & $05 t$ & $01 C \backsim 8$ & 1 & 189 & 01 & 15 & 75 & & & & 370 & 85.0 & 210.0 & 02.0 & 1.00 & \\
\hline $10 \mathrm{~N}$ & OGE & $17 A D D$ & 1 & 25 & 01 & 29 & 70 & & 8 & 11.0 & 154 & 4.8 & 8.0 & 2.7 & & 197 \\
\hline $10 \mathrm{~N}$ & $07 \mathrm{~F}$ & $1 B B C C$ & 1 & 42 & 05 & 04 & 70 & & & 11.0 & 20 & 24.0 & 13.0 & 15.0 & & 113 \\
\hline $11 \mathrm{~N}$ & OGE & $34 B C C$ & 1 & 198 & 08 & 11 & 70 & & & 134.0 & 248 & 163.0 & 11.0 & 0.1 & & 478 \\
\hline $11 \mathrm{~N}$ & $07 E$ & $35 A A C$ & $i$ & 152 & 05 & 21 & 70 & & & 45.0 & 324 & 57.0 & 14.0 & 0.4 & & 374 \\
\hline
\end{tabular}

PUTTAWATOMIE COUNTY

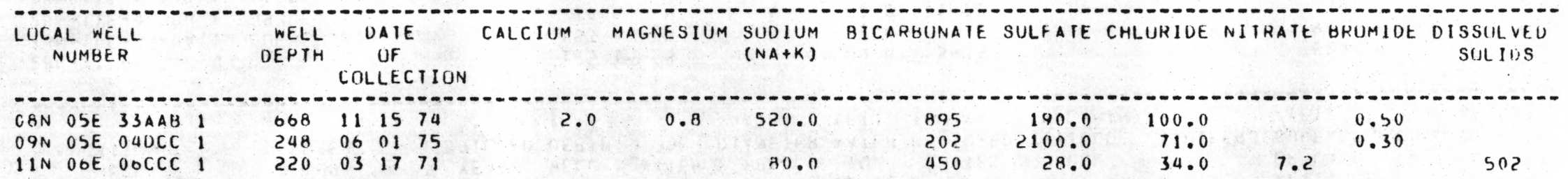

OKFUSKEE COUNTY

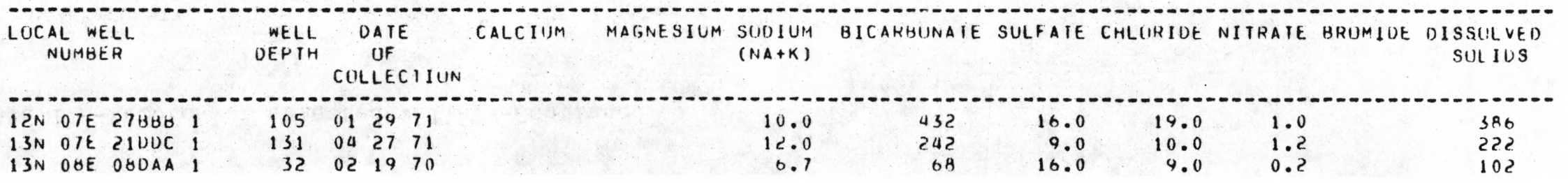


Table 2.--Chemical analyses of water from selected we1ls.--Continued

LINCOLN CUUNTY

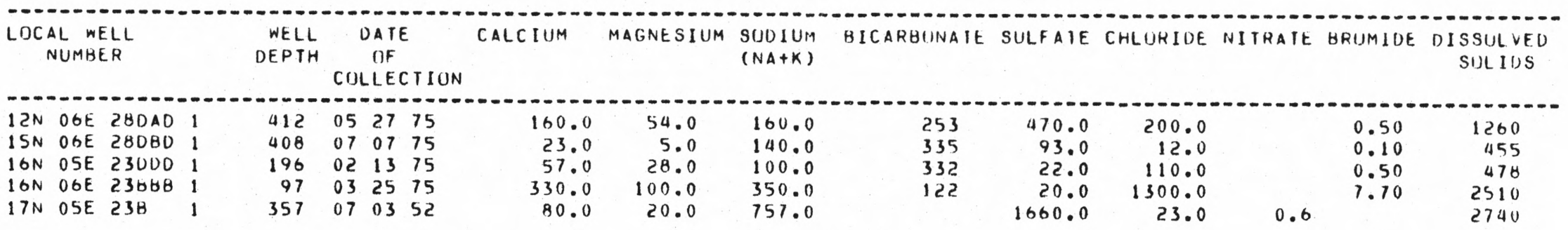

CREEK CUUNTY

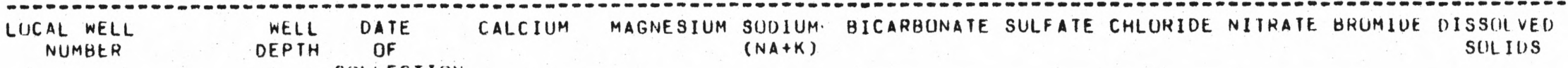
COLLECTION

\begin{tabular}{|c|c|c|c|c|c|c|c|c|c|c|c|c|c|c|c|c|}
\hline $14 N$ & $07 E$ & $10 \mathrm{CBB}$ & 1 & 153 & 04 & 27 & 71 & & & 25.0 & 374 & 7.0 & 100.0 & 0.5 & & 486 \\
\hline $14 \mathrm{~N}$ & $08 E$ & OUAAC & 1 & 55 & 11 & 06 & 70 & & & 30.0 & 76 & 15.0 & 30.0 & 4.0 & & 146 \\
\hline $15 \mathrm{~N}$ & $07 E$ & $25 C C C$ & 1 & 149 & 10 & 11 & 74 & 12.0 & 15.0 & 13.0 & 217 & 2.8 & 6.9 & & 0.10 & 201 \\
\hline $15 \mathrm{~N}$ & OBE & $06 \mathrm{COB}$ & 1 & 124 & 06 & 11 & 71 & & & 12.0 & 254 & 11.0 & 7.0 & 0.4 & & 243 \\
\hline $16 N$ & OTE & $21 A D A$ & 1 & 117 & 06 & 11 & 71 & & & 4.9 & 46 & 11.0 & 9.0 & 0.8 & & 77 \\
\hline $16 \mathrm{~N}$ & $07 E$ & $3300 A$ & 1 & 99 & 06 & 10 & 71 & & & 26.0 & 306 & 24.0 & 16.0 & 2.2 & & 324 \\
\hline $17 N$ & $07 E$ & OBCCA & $i$ & 487 & 07 & 23 & 71 & & & 110.0 & 302 & 28.0 & 45.0 & 0.1 & & 388 \\
\hline $17 \mathrm{~N}$ & OTE & 25400 & 1 & 130 & 04 & 23 & 71 & & & 17.0 & 108 & 14.0 & 13.0 & 0.3 & & 134 \\
\hline $17 \mathrm{~N}$ & $08 E$ & $O \triangle A C C$ & 1 & 136 & 06 & 15 & 71 & & & 25.0 & 240 & 4.4 & 7.0 & 0.8 & & 235 \\
\hline $17 N$ & OOE & $3580 \mathrm{~A}$ & $i$ & 121 & 01 & 29 & 75 & 19.0 & 8.5 & 8.8 & 76 & 11.0 & 16.0 & 0.2 & 0.02 & \\
\hline $18 N$ & $O 6 E$ & $360 A D$ & 1 & 538 & 10 & 30 & 75 & 11.0 & 1.2 & 140.0 & 273 & 79.0 & 16.0 & & 0.10 & 405 \\
\hline $18 \mathrm{~N}$ & $O D E$ & 36000 & 1 & 490 & 07 & 23 & 71 & & & 66.0 & 256 & 63.0 & 12.0 & 0.0 & & 340 \\
\hline $18 \mathrm{~N}$ & $07 E$ & $13 A A O$ & 1 & 77 & 12 & 14 & 71 & & & 5.2 & 92 & 22.0 & 8.6 & 1.6 & & 142 \\
\hline $18 \mathrm{~N}$ & $07 E$ & 16BAD & 1 & 600 & 04 & 01 & 75 & 19.0 & 1.9 & 190.0 & 266 & 180.0 & 49.0 & & 0.20 & 595 \\
\hline $18 \mathrm{~N}$ & $07 E$ & $20 A$ & 1 & 124 & 06 & 08 & 71 & & & 29.0 & 266 & 110.0 & 52.0 & 0.7 & & 480 \\
\hline $18 \mathrm{~N}$ & $07 t$ & 3500A & 1 & 230 & 06 & 08 & 71 & & & 25.0 & 390 & 30.0 & 13.0 & 0.4 & & 358 \\
\hline $18 \mathrm{~N}$ & $O B E$ & $1 \triangle B C C$ & 1 & 120 & 11 & 14 & 72 & 40.0 & 16.0 & 17.0 & 232 & 11.0 & 5.0 & 0.0 & & 207 \\
\hline $18 \mathrm{~N}$ & $08 E$ & 33000 & 1 & 194 & 01 & 29 & 75 & 7.0 & 3.1 & 11.0 & 12 & 8.6 & 17.0 & 0.1 & 0.02 & $8 H$ \\
\hline $19 \mathrm{~N}$ & $07 E$ & $03 C D D$ & 1 & 160 & 02 & 23 & 73 & 110.0 & 72.0 & 130.0 & 486 & 180.0 & 140.0 & 83.0 & & 960 \\
\hline $19 \mathrm{~N}$ & $0 B E$ & $040 C C$ & 1 & 67 & 02 & 23 & 73 & 14.0 & 4.6 & 23.0 & 76 & 13.0 & 5.4 & 27.0 & & 132 \\
\hline
\end{tabular}


Table 2.--Chemical analyses of water from selected wells.--Continued

payne cuunty

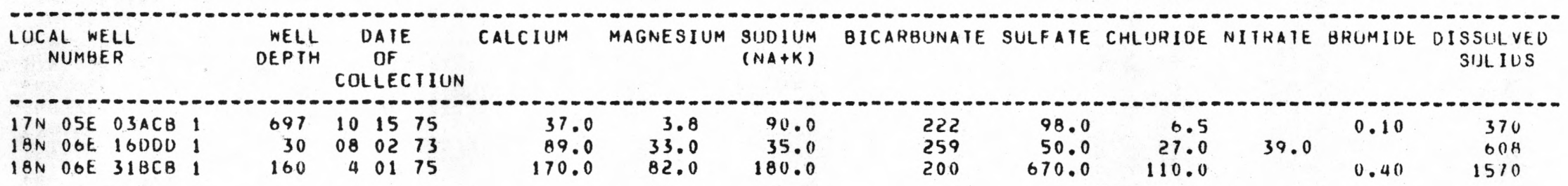

PAWNEE COUNIY

\begin{tabular}{|c|c|c|c|c|c|c|c|c|c|c|c|c|c|c|c|c|}
\hline \multicolumn{4}{|c|}{$\begin{array}{l}\text { LOCAL WELL } \\
\text { NUMBER }\end{array}$} & $\begin{array}{l}\text { WELL } \\
\text { DEPTH }\end{array}$ & & $\begin{array}{l}\text { DATE } \\
\text { OF } \\
\text { LLEC }\end{array}$ & $\begin{array}{l}\text { E } \\
\text { CTION }\end{array}$ & CALCIUM & MAGNESIUM & $\begin{array}{l}\text { SUDIUM } \\
(N A+K)\end{array}$ & BICARBONATE & SULFATE & CHLURIDE & NIIRAIE & BRUMIDE & $\begin{array}{l}\text { OISSULVED } \\
\text { SULIISS }\end{array}$ \\
\hline $18 \mathrm{~N}$ & OOE & $31 \mathrm{BC}$ & 1 & & 04 & 01 & 75 & 170.0 & 82.0 & & & & & & & \\
\hline $20 N$ & $07 E$ & OIDAD & 1 & 90 & 04 & 09 & 75 & 390.0 & 130.0 & 110.0 & 413 & 970.0 & 270.0 & & 0.70 & 2220 \\
\hline $20 N$ & $08 E$ & OSOUA & 1 & 28 & 08 & 02 & 71 & & & 34.0 & 24 & 12.0 & 110.0 & & & 234 \\
\hline $20 N$ & $08 E$ & $06 C C C$ & 1 & & O4 & 10 & 75 & 140.0 & 110.0 & 74.0 & 487 & 450.0 & BO. 0 & & 0.30 & 1200 \\
\hline $20 \mathrm{~N}$ & $O B E$ & $090 C 0$ & 1 & 20 & 08 & 02 & 71 & & & 81.0 & 44 & 66.0 & 220.0 & 26.0 & & 676 \\
\hline $20 N$ & $08 E$ & $17 \mathrm{AAD}$ & 1 & 80 & 08 & 02 & 71 & & & 150.0 & 140 & 250.0 & 150.0 & & & 744 \\
\hline $21 \mathrm{~N}$ & $O H E$ & 2ODCA & 1 & 85 & 08 & 13 & 71 & & & 32.0 & 72 & 91.0 & 90.0 & 4.2 & & 382 \\
\hline $21 \mathrm{~N}$ & $08 E$ & 29AAD & 1 & 65 & 08 & 19 & 71 & & & 102.0 & 50 & 58.0 & 360.0 & 20.0 & & 998 \\
\hline $22 N$ & $07 E$ & $27 A B B$ & 1 & & 10 & 01 & 73 & 118.0 & 23.0 & 30.0 & 389 & 46.0 & 32.0 & 11.0 & & 488 \\
\hline
\end{tabular}


Table 2.--Chemical analyses of water from selected wells.--Continued

osage cuUnty

\begin{tabular}{|c|c|c|c|c|c|c|c|c|c|c|c|c|c|c|c|c|}
\hline \multicolumn{4}{|c|}{$\begin{array}{c}\text { LUCAL WELL } \\
\text { NUMBER }\end{array}$} & $\begin{array}{r}\text { WELL } \\
\text { DEPTH }\end{array}$ & \multicolumn{3}{|c|}{$\begin{array}{l}\text { DATE } \\
\text { OF }\end{array}$} & CALCIUM & MAGNESIUM & $\begin{array}{l}\text { SUD IUM } \\
(N A+K)\end{array}$ & BICARBUNATE & SULFATE & CHLURIOE & NITRATE & BRUMIOE & $\begin{array}{c}\text { DISSUL VED } \\
\text { SUL IDS }\end{array}$ \\
\hline $21 \mathrm{~N}$ & $07 E$ & $11 C A A$ & 1 & 42 & 07 & 11 & 73 & 30.0 & 5.6 & 13.0 & 108 & 13.0 & 7.8 & 4.9 & & 192 \\
\hline $22 N$ & $07 E$ & 10080 & 1 & 21 & 07 & 11 & 73 & 110.0 & 28.0 & 64.0 & 372 & 6.0 & 68.0 & 17.0 & & 712 \\
\hline $22 \mathrm{~N}$ & $08 E$ & 3SBAA & 1 & 100 & 08 & 16 & 71 & & & 72.0 & 376 & 28.0 & 31.0 & 7.0 & & 408 \\
\hline $22 \mathrm{~N}$ & $09 E$ & $17 \mathrm{CCC}$ & 1 & 200 & 01 & 18 & 73 & 6.0 & 8.0 & 180.0 & 416 & 38.0 & 25.0 & 0.4 & & 466 \\
\hline $23 N$ & OTE & $0980 A$ & 1 & 87 & 01 & 18 & 73 & 22.0 & 13.0 & 140.0 & 386 & 59.0 & 16.0 & 2.4 & & 454 \\
\hline $23 N$ & $08 \mathrm{E}$ & $070 C C$ & 1 & 350 & 04 & 04 & 75 & 220.0 & 57.0 & 140.0 & 176 & 120.0 & 570.0 & & 3.30 & 1410 \\
\hline $23 N$ & $09 E$ & $25 B C C$ & 1 & 187 & 04 & 03 & 75 & 87.0 & 60.0 & 77.0 & 205 & 190.0 & 110.0 & & 0.60 & 708 \\
\hline $24 N$ & OBE & $33 C C A$ & 1 & 25 & 08 & 20 & 71 & & & 40.0 & 388 & 120.0 & 28.0 & 12.0 & & 560 \\
\hline $24 N$ & $09 E$ & OSOAA & 1 & 260 & 04 & 02 & 75 & 5.1 & 3.1 & 240.0 & 420 & 53.0 & 54.0 & & 0.30 & 627 \\
\hline $24 N$ & OYE & $20 A B B$ & 1 & 325 & 08 & 19 & 71 & & & 53.0 & 192 & 37.0 & 11.0 & 0.5 & & 250 \\
\hline $25 \mathrm{~N}$ & OSE & $190 C B$ & 1 & & 04 & 03 & 75 & 180.0 & 41.0 & 150.0 & 253 & 24.0 & 470.0 & & 2.0 .0 & 1180 \\
\hline $25 N$ & $09 E$ & $24 B A 0$ & 1 & 240 & 11 & 16 & 72 & 33.0 & 28.0 & 160.0 & 480 & 86.0 & 43.0 & 1.0 & & 582 \\
\hline $25 N$ & $09 E$ & 35BAD & 2 & 255 & 02 & 22 & 73 & 120.0 & 34.0 & 61.0 & 262 & 150.0 & 110.0 & 58.0 & & 658 \\
\hline $26 \mathrm{~N}$ & $09 E$ & $14 \triangle C B$ & 1 & 109 & 04 & 10 & 73 & 19.0 & 7.8 & 19.0 & 37 & 7.6 & 39.0 & & & \\
\hline $26 \mathrm{~N}$ & $10 E$ & OQAUA & 1 & 130 & 03 & 13 & 75 & 11.0 & 2.8 & 600.0 & 447 & & 640.0 & & 3.80 & 1490 \\
\hline $27 N$ & O9E & $27 A C C$ & 1 & 125 & 02 & 22 & 73 & 4.0 & 1.2 & 270.0 & 590 & 94.0 & 18.0 & 0.4 & & 670 \\
\hline $28 \mathrm{~N}$ & OTE & 2900A & 1 & 900 & 03 & 26 & 75 & 8.1 & 3.4 & 860.0 & 560 & 220.0 & 860.0 & & 4.50 & 2290 \\
\hline $28 \mathrm{~N}$ & $08 E$ & OSAAA & 1 & 300 & 04 & 10 & 73 & 8.7 & 1.8 & 19.0 & 378 & 40.0 & 110.0 & 12.0 & & 637 \\
\hline $28 \mathrm{~N}$ & $08 E$ & OBODA & 1 & 500 & 03 & 06 & 75 & 4.7 & 2.0 & 440.0 & 411 & 20.0 & 440.0 & & 0.10 & 1140 \\
\hline $28 \mathrm{~N}$ & $O 3 E$ & $150 A C$ & 1 & 160 & 03 & 12 & 75 & 1.8 & 0.1 & 210.0 & 386 & 56.0 & 63.0 & & 0.40 & 542 \\
\hline $28 \mathrm{~N}$ & $09 E$ & $2900 A$ & 1 & 900 & 03 & 26 & 75 & 8.1 & 3.4 & 860.0 & 560 & 220.0 & 860.0 & & 4.50 & 2290 \\
\hline $28 \mathrm{~N}$ & $10 E$ & S3ADA & 1 & 153 & 01 & 17 & 73 & 42.0 & 14.0 & 17.0 & 158 & 40.0 & 20.0 & 7.5 & & 235 \\
\hline $29 N$ & $09 E$ & $23 C D A$ & 1 & 90 & 03 & 06 & 75 & 99.0 & 31.0 & 150.0 & 336 & 45.0 & 280.0 & & 1.80 & 1030 \\
\hline $29 \mathrm{~N}$ & $10 E$ & $29 B C A$ & 1 & 125 & 11 & 20 & 73 & & & & & & 1000.0 & & 5.00 & \\
\hline $29 N$ & $10 E$ & $2960 \mathrm{C}$ & 1 & 65 & 01 & 31 & 74 & 140.0 & 14.0 & 24.0 & 304 & 34.0 & 120.0 & & 0.55 & 533 \\
\hline $29 N$ & $10 E$ & $29 \forall D C$ & 1 & & 01 & 31 & 73 & 140.0 & 14.0 & 25.0 & 317 & 36.0 & 120.0 & & 0.85 & 568 \\
\hline $29 \mathrm{~N}$ & IOE & 29CAA & 1 & & 01 & 31 & 74 & 1400.0 & 200.0 & 2500.0 & & 120.0 & 9400.0 & & 9.99 & 14300 \\
\hline
\end{tabular}


Table 3.--Stream site data.

SEMINULE COUNTY

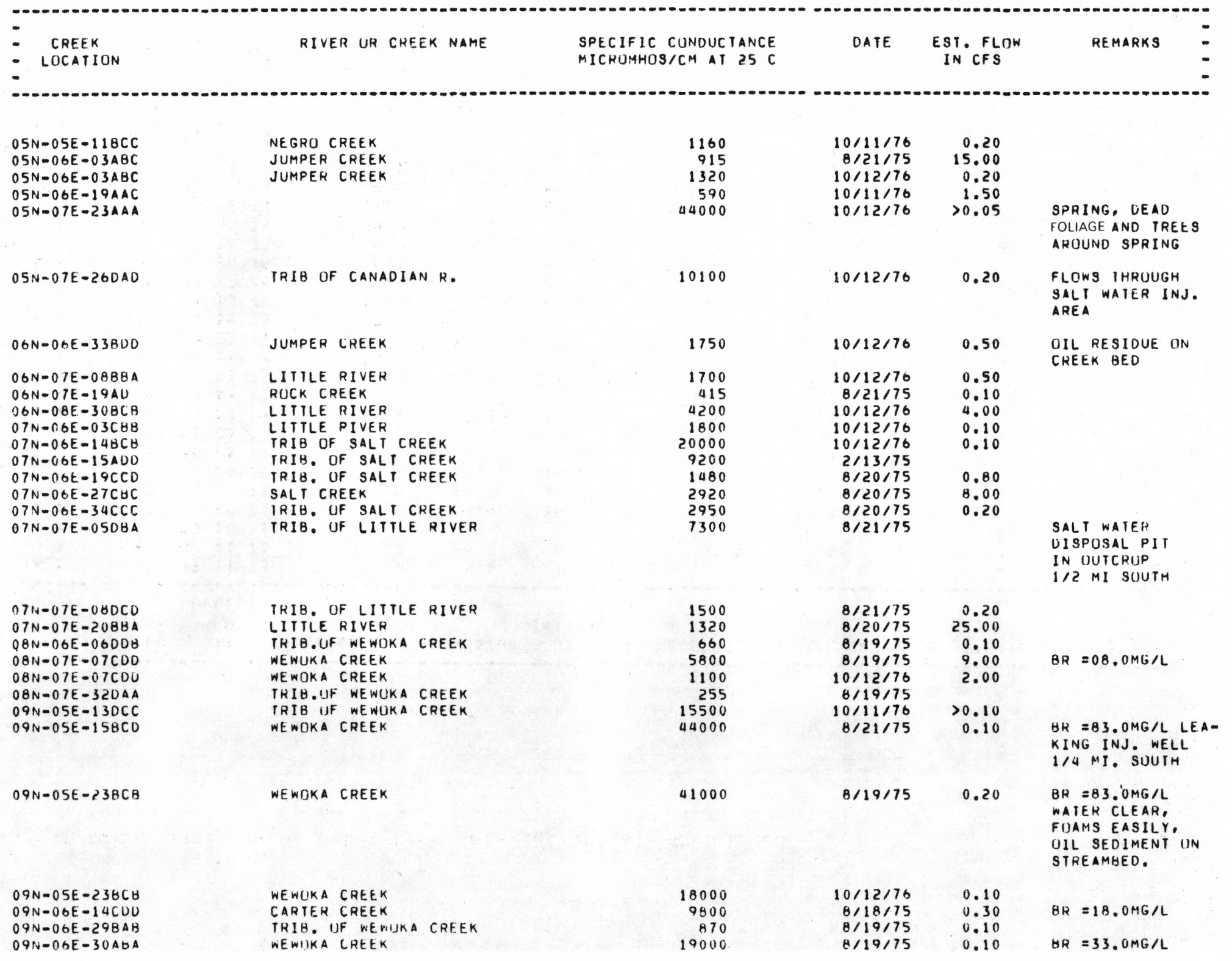


Table 3.--Stream site data.--Continued

SEMINOLE COUNTY

CREEK
- LOCATION

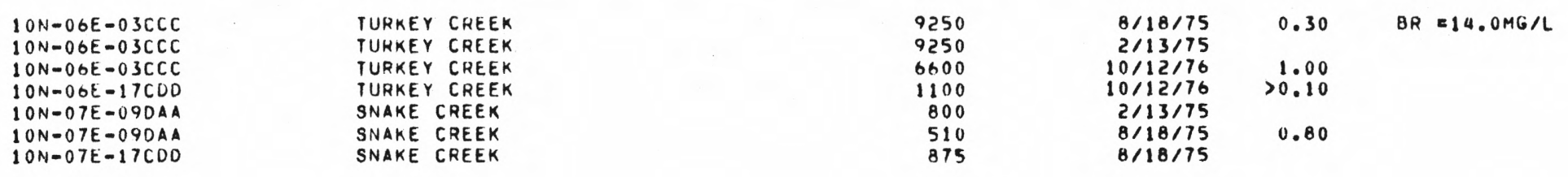

POTTAWATUMIE COUNTY

\begin{tabular}{|c|c|c|c|c|c|}
\hline $\begin{array}{l}-\quad \text { CREEK } \\
-\quad \text { LOCATION }\end{array}$ & RIVER OR CREEK NAME & $\begin{array}{l}\text { SPECIFIC CUNDUCTANCE } \\
\text { MICRUMHUS/CM AT } 25 \mathrm{C}\end{array}$ & DATE & $\begin{array}{l}\text { EST. FLUW } \\
\text { IN CFS }\end{array}$ & REMARKS \\
\hline $\begin{array}{l}11 N-05 E-110 C D \\
11 N-06 E-168 A D \\
11 N-08 E-13 A D D\end{array}$ & $\begin{array}{l}\text { TRIB. OF N. CANADIAN RIVER } \\
\text { SHAN CREEK } \\
\text { TRIB UF N. CANAIDAN R. }\end{array}$ & $\begin{array}{l}620 \\
590 \\
575\end{array}$ & $\begin{array}{r}0 / 18 / 75 \\
8 / 19 / 75 \\
10 / 12 / 76\end{array}$ & $\begin{array}{l}0.10 \\
0.20 \\
0.10\end{array}$ & \\
\hline
\end{tabular}

DKFUSKEE COUNIY

CREEK
- LOCATIUN RIVER OR CREEK NAME

1 IN-07E-05BBA $11 N-0 B E-13 \triangle 00$ $11 \mathrm{~N}-08 \mathrm{E}-14 \mathrm{AAA}$ $12 N-07 E-06 B 8 A$ $12 N-07 E-33 C C D$ $12 N-08 E-358 B C$ $12 N-0 B E-35 B B C$

$13 N-07 E-31 A B B$ $13 N-07 E-02 D C U$ $13 N-08 E-01 C C D$ I $3 N-09 E-06 A A D$
TRIB. UF NURIH FORK CREEK N. CANAUIAN RIVER IRIB. OF N. CANADIAN R. PETTIQUAH CREEK

TRIQ. OF NIJRTH FORK CREEK

TRIB. OF NORTH FORK CREEK

TRIB UF N. CANAUIAN R.

PETIIIUUAH CREEK

HILLIBY CREEK

IRIB. OF DEEP FURK OF CANADIAN H

DEEP FURK UF CANADIAN R.

660
1850
220
270
670
1300
101000
1190
625
370
1220

$2 / 14 / 75$
$10 / 12 / 76$
$10 / 12 / 76$
$8 / 18 / 75$
$2 / 14 / 75$
$2 / 17 / 75$
$10 / 12 / 76$
$1 / 30 / 75$
$8 / 22 / 75$
$10 / 13 / 76$
$10 / 13 / 76$

80.00

1.00
3.00

$>0.10$

1.00

1.00
0.10

70.00
LEAK FROM INJ. AHEA 
Table 3.--Stream site data.--Continued

LINCOLN COUNTY

CREEK
- LOCATIUN RIVER OR CREEK NAME

\section{$12 N-05 E-020 A D$ $12 N-06 E-03 B 8 A$ \\ $12 N-06 E-03 B 8 A$ \\ $12 \mathrm{~N}-06 \mathrm{O}-10 \mathrm{CCC}$ \\ $13 \mathrm{~N}-06 \mathrm{C}-040 \mathrm{CC}$ \\ $13 N-06 E-09 C C C$ \\ $13 N-06 E-11 A B B$ \\ $13 N-06 E-21 A B A$ \\ $14 N-06 E-0302 C$ \\ $14 N-06 E-030 C C$ \\ $14 \mathrm{~N}-06 \mathrm{CE}-15 \mathrm{CBB}$ \\ $14 \mathrm{~N}-06 \mathrm{E}-34 \mathrm{AAB}$}

$14 N-06 E-36 C D 0$

$14 N-06 E-36 C D D$

$15 N-06 E-228 B B$

$16 N-05 E-01 A A D$

$16 N-05 E-36 A A A$

$16 N-06 E-06 C D O$

$16 N-06 E-06 C 00$

$16 N=06 E-06 C 0 D$

$17 N-06 E-180 C C$

$17 N-06 E-180 C C$
$17 N-06 E-19 C O C$

SPRING CREEK
DEER CREEK
DEER CREEK
DEER CEEEK
DEER CREEK
DEER CREEK
TRIB. OF DEEP FURK DF CANADIAN
DEER CREEK
TRIB. OF DEEP FORK OF CANADIAN
TRIB. OF DEEP FURK OF CANAUIAN
DEEP FURK OF CANADIAN RIVER
TRIB. OF DEEP FURK OF CANADIAN

TRIB, OF DEEP FURK OF CANADIAN

TRIB. OF DEEP FORK OF CANADIAN

IRIB. OF DEEP FIIRK OF CANADIAN

SALT CREEK

IRIB. OF EUCHEE CREEK

FOURMILE CREEK

EUCHEE

EUCHEE

EUCHEE

EUCHEE

EUCHEE
1480

900
346

346
610

940

730

1090

995

4600

1120

1300

$2 / 14 / 75$

$2 / 14 / 75$

$8 / 18 / 75$

$8 / 18 / 75$

$2 / 14 / 75$

$8 / 18 / 75$

$1 / 30 / 75$

2112175

$1 / 30175$

$1 / 30175$

$8 / 19 / 75$

1.50

0.40

6.00

(8)

$B R=02.2 M G / L$

OIL LINING

CREEK BANK

$2 / 12 / 75$

$8 / 19 / 75$

$6 / 19 / 75 \quad 4.00$

$8 / 19 / 75 \quad 5.00$

$2 / 13 / 75$

रा12/75

2112175

$8 / 19 / 75$

2113175

$8 / 20 / 75$

0.50

210

2600
245

245
220

PAYNE COUNTY

CREEK
LUCATION


Table 3.--Stream site data.--Continued

CREEK COUNTY

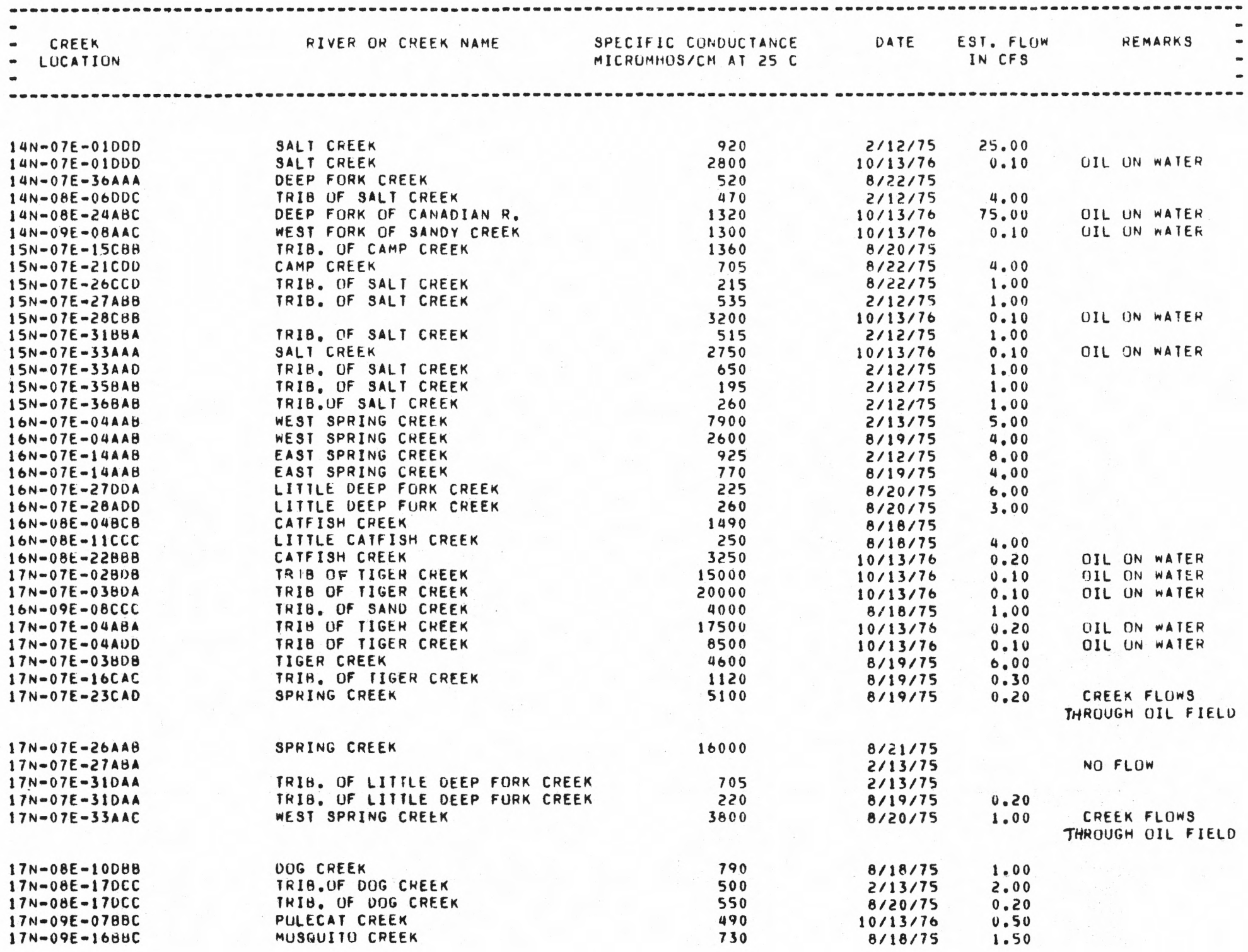


Table 3.--Stream site data.--Continued

CREEK COUNTY

- CREEK
- LUCATIUN RIVER UR CREEK NAME

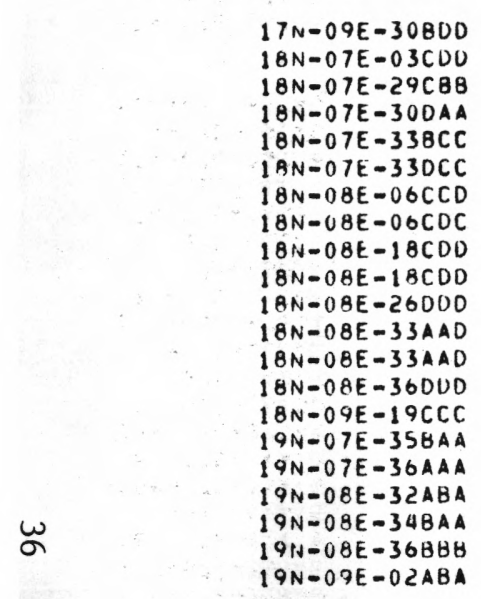

MUSQUITO CREEK

DRY CREEK

TRIB OF TIGER CREEK

TIGER CREEK

TRIB. OF TIGER CREEK

TIGER CREEK

BUCKEYE CREEK

DUCKEYE CREEK

BUCKEYE CREEK

BUCKEYE CREEK

BUCKEYE CREEK

DEEP CREEK

PULECAT CREEK
TRIB. OF POLECAT CREEK

FIGURE EIGHT CREEK

FIGURE EIGHT CRFEK

DKY CREEK

QUCKEYE CREEK

SAND CREEK

ROCK CANYON CREEK

COITONWOOD CREEK

LITTLE SALT CREEK

$\begin{array}{rrr}1300 & 10 / 13 / 76 & 0.50 \\ 3100 & 8 / 20 / 75 & 1.00 \\ 4180 & 10 / 13 / 76 & 1.50 \\ 12100 & 10 / 13 / 76 & 0.20 \\ 11400 & 10 / 13 / 76 & 0.30 \\ 14000 & 10 / 13 / 76 & 0.20 \\ 260 & 8 / 19 / 75 & 4.00 \\ 420 & 10 / 13 / 76 & 1.00 \\ 270 & 8 / 10 / 75 & \\ 275 & 10 / 13 / 76 & 1.00 \\ 220 & 8 / 18 / 75 & 2.00 \\ 310 & 8 / 19 / 75 & 5.00 \\ 220 & 10 / 13 / 76 & 1.00 \\ 250 & 8 / 18 / 75 & 0.80 \\ 160 & 10 / 13 / 76 & 0.20 \\ 1450 & 8 / 20 / 75 & 2.00 \\ 500 & 10 / 13 / 76 & 2.00 \\ 330 & 8 / 20 / 75 & 0.80 \\ 230 & 8 / 20 / 75 & 2.00 \\ 470 & 8 / 19 / 75 & 1.50 \\ 410 & 8 / 21 / 75 & 0.10\end{array}$

OIL ON WATER

$B R=03.8 \mathrm{MG} / \mathrm{L}$

OIL UN WATER

OIL ON WATER

OIL UN WATER

NUT BASE FLUW

CREEK FLUWS

THRUUGH UIL FIELD

PAWNEE CUUNTY

\begin{tabular}{|c|c|c|}
\hline $\begin{array}{l}-\quad \text { CREEK } \\
-\quad \text { LOCATION }\end{array}$ & RIVER OR CREEK NAME & $\begin{array}{l}\text { SPECIFIC CONDUCT } \\
\text { MICROMHUS/CM AT }\end{array}$ \\
\hline $\begin{array}{l}20 N-08 E-020 A C \\
20 N-08 E-020 A A \\
20 N-08 E-06000 \\
20 N-08 E-13 B 0 A \\
20 N-08 E-32 A 6 B \\
21 N-07 E-21 B B C \\
21 N-07 E-216 B C \\
21 N-08 E-17 B C B \\
21 N-08 E-17 B C B\end{array}$ & $\begin{array}{l}\text { FEYOOI CREEK } \\
\text { FEYUDI CREEK } \\
\text { TRIB. OF HUUSE CREEK } \\
\text { CUWSKIN CREEK } \\
\text { TRIB. UF HOUSE CREEK } \\
\text { TURKEY CREEK } \\
\text { TURKEY CHEEK } \\
\text { CEDAR CREEK } \\
\text { CEUAR CREEK }\end{array}$ & $\begin{array}{l}2300 \\
1160 \\
4050 \\
3100 \\
350 \\
310 \\
360 \\
4150 \\
4650\end{array}$ \\
\hline
\end{tabular}

DATE

ESI. FLOWW

IN CFS

REMARKS

IN CFS

$\begin{array}{rrr}00 & 8 / 21 / 75 & 1.00 \\ 60 & 10 / 14 / 76 & 0.10 \\ 50 & 8 / 21 / 75 & 1.00 \\ 00 & 8 / 21 / 75 & 0.10 \\ 50 & 8 / 21 / 75 & 2.00 \\ 10 & 8 / 21 / 75 & \\ 60 & 10 / 13 / 76 & 0.10 \\ 50 & 8 / 21 / 75 & 3.00 \\ 50 & 10 / 13 / 76 & 0.50\end{array}$

$\theta R=06.7 \mathrm{MG} / \mathrm{L}$

$B R=05.8 \mathrm{MG} / \mathrm{L}$

OIL ON WATER 
Table' 3.--Stream site data.--Continued

DSAGE COUNTY

- CREEK
- LOCATIUN RIVER UR CREEK NAME

$21 N-10 E-04 C D D$ $22 N-09 E-13 C 0 D$ $22 N-09 E-16 A D B$ $22 N-10 E-20 B D D$ $22 N-10 E-21 A B D$ $2 Z N-10 E-290 C$ $22 N-10 E-3200 C$ $23 N-08 E-05 C B E$ $23 N-08 E-35 C D A$ $23 N-09 E-180 B C$ $23 N-09 E-27 A 0 A$ $23 \mathrm{~N}-09 \mathrm{E}-30 \mathrm{ADD}$ $23 N=09 E-330 A C$ $23 N-0 O E-34 A D D$ $23 N-09 E-36 D A A$ $23 N-10 E-34 C D D$ I $A N-07 E-10 D C A$ $24 N-07 E-100 C A$ 24N-07E-33AAA $24 N-07 E-33 C C C$ $24 N-09 E-09 A C C$ $24 N-08 E-14 C B G$ $24 N-08 E-14 C B B$ $24 N-0 B E-258 A D$ $2 \triangle N-O B E-33$ $24 N-09 E-32 D 0 B$ 2 $4 N-D$ CE- $3200 B$ $24 N=O B E-36 C D O$ $24 N-10 E-0100 B$ $24 N-10 E-170 A C$ $24 N-10 E-170 A 0$ $24 N-10 E-30 C A A$ $25 N-0 B E-18$ $25 N-08 E-19 C A B$ $25 N-08 E-19 C C D$

$25 N-08 E-23800$ $25 \mathrm{~N}-08 \mathrm{E}-308 \mathrm{~B} B$ $25 N-10 E-028 B B$ $25 N-10 E-14 C C C$
TRIB OJF WILO HORSE CREEK

BUAR CKEEK

HUMINY CREEK

WILD HURSE CREEK

EAGLE CREEK

BUCK CREEK

WILDHURSE CREEK

TRIB. OF HELLROARING CREEK

PEEN CREEK

OIG HOMINY CREEK

SUNSET CREEK

TRIB. UF TWUMILE CREEK

BULL CREEK

HOMINY CRE

SCYAMURE CREEK

SYCAMORE CREEK

LITTLE HOMINY CREEK

LITILE HOMINY CREEK

LITILE ROMINY CREEK

TURK EYRUN CREEK

LITTLE HOHINY CREEK

RED EAGLE BRANCH

BURCH CREEK

BERCH CREEK

FUURMILE CREEK

CLEAR CREEK

TRIB. OF LITTLE HOMINY CREEK

TRIB.UF LITILE HUMINY CREEK

CLEAR CREEK

CLEAR CREEK

PUNO CREEK

CLEAR CKEEK

NELAGUNEY CREEK
$10 / 14 / 76 \quad 0.10$

$10 / 14 / 76 \quad 2.00$

$10 / 14 / 76 \quad 5.00$

$10 / 14 / 76 \quad 0.50$

$10 / 14 / 76 \quad 0.30$

$\begin{array}{ll}10 / 14 / 76 & 0.50 \\ 10 / 14 / 76 & 0.30\end{array}$

$\begin{array}{ll}10 / 14 / 76 & 0.30 \\ 10 / 14 / 76 & 0.10\end{array}$

$10 / 14 / 76 \quad 0.50$

$10 / 14 / 76 \quad 10.00$

$10 / 14 / 76 \quad 1.00$

$8 / 18 / 75$

$10 / 14 / 76$

$10 / 14 / 76$

$10 / 14 / 76$

$10 / 14 / 76$

$10 / 14 / 76$

$8 / 18 / 75$

$8 / 18 / 75$

$10 / 14 / 76$

$10 / 14 / 76$

$8 / 18 / 75$

$10 / 14 / 76$

$10 / 14 / 76$

$8 / 18 / 75$

$10 / 14 / 76$

$8 / 18 / 75$

$10 / 14 / 76$

$6 / 20 / 75$

$10 / 14 / 76$

$8 / 18 / 75$

$8 / 19 / 75$

1.00

1.00

1.00

0.20

4.00

0.20

1.00

5.00

0.10

2.00

4.00

0.10

0.10

10.00

0.20

0.10

2.00

42000

NIIT BASE FLIJW

NOT BASE FLOW

$B R=31.0 M G / L$

$8 / 19 / 75$

$6 / 18 / 75$

$10 / 14 / 76$
$8 / 20 / 75$

10.00

1225
520

190

$8 / 20175$

$8 / 20 / 75$
NOT BASE FLOW

$B R=18.0 \mathrm{MG} / \mathrm{L}$

BRINE FLOWING

INTU CREEK FROM

BHINE PII.

$B R=70.0 \mathrm{MG} / \mathrm{L}$

NOT BASE FLUW EVIUENCE OF OIL RELEASE ALONG STREAM BANK

NUT BASE FLUN 
Table 3.--Stream site data.--Continued

osage countr

CREEK
LUCATION RIVER UR CREEK NAME

$25 N-10 E-18 B C C$

$25 N-10 E-200 B B$

$25 N-10 E-218 C B$

$25 N-10 E-21 B C H$

$25 N-10 E-3200 C$

2BN-09E-12AAA

$26 N=10 E-04 C A D$

$26 N-10 E-15 A 4 A$

$26 N-10 E-19 C A A$

$27 \mathrm{~N}-10 \mathrm{E}-$

$27 N-10 E-07 C B G$

$27 N-10 E-0 B C A A$

$27 N=10 E-0 B C D D$

$27 N-10 E-30 C C B$

$28 N-0 B E-03 A Q A$

$39 N-08 E-13 C B Q$

$29 N-0 B F-220 A A$

$29 N-09 E-13 D A B$

$59 N-09 E-230 C A$

$29 N-09 E-23000$

$29 N-10 E-16 A B B$

$29 N-10 E-17 A A B$

$29 N-10 E-170 A C$

$29 N-11 E-18000$

$29 N-11 E-18000$

$29 N-11 E-30 A B A$

$29 N-11 E-30 A B A$

$29 N-11 E-30 A B A$
QUAPAW CREEK

CAT CREEK

BUFFALU CREEK

BUFFALO CREEK

CEDAR CREEK

ROCK CREEK

SAND CREEK

SAND CREEK

TRIB. UF ROCK CREEK

TRIB. OF ROCK CREEK

TRIB. UF RUCK CREEK

HOCK CREEK

ELM CREEK

BUCK CREEK

SMITH CREEK

SMITH CREEK

CANEY RIVER

BUCK CREEK

BUCK CREEK

CANEY RIVER

TRIB. OF CANEY RIVER

CANEY RIVER

TURKEY CREEK

TURKEY CREEK

TURKEY CREEK

TURKEY

TURKEY CREEK

TURKEY CFEEK

2650
810
480
1860
420
640
600
700
420
430
340
590
260
340
220
420
350
395
450
660
400
460
120
2900
3500
1200
4800

3500

$8 / 20 / 75$

$10 / 14 / 76$

$3 / 26 / 75$

$3 / 26 / 75$

10714776

$8 / 20175$

$8 / 20 / 75$

$8 / 20 / 75$

$8 / 20 / 75$

$8 / 21 / 75$

$8 / 21 / 75$

$8 / 21 / 75$

$8 / 21 / 75$

$8 / 21 / 75$

$3 / 06 / 75$

$8 / 21 / 75$

$3 / 00 / 75$

$8 / 21 / 75$

$3 / 06 / 75$

$8 / 21 / 75$

$3 / 06 / 75$

$3 / 06 / 75$

$3 / 26 / 75$

$9 / 21 / 75$

$10 / 14 / 76$

$3 / 06 / 75$

$8 / 21 / 75$

$10 / 14 / 70$
1.00

20.00

0.50

11.00

0.20

0.40

0.20

0.20

0.40

0.20

0.20

0.50

2.00

0.10
UIL ON WATER

NOT BASE FLOW

NUT BASE FLUW

NOT BASE FLUW

OIL DN WATER

$B R=12.0 \mathrm{MG} / \mathrm{L}$ UIL ON WATER 